\title{
Peroxiredoxins and Redox Signaling in Plants
}

\author{
Michael Liebthal, Daniel Maynard, and Karl-Josef Dietz
}

\begin{abstract}
Significance: Peroxiredoxins (Prxs) are thiol peroxidases with multiple functions in the antioxidant defense and redox signaling network of the cell. Our progressing understanding assigns both local and global significance to plant Prxs, which are grouped in four Prx types. In plants they are localized to the cytosol, mitochondrion, plastid, and nucleus. Antioxidant defense is fundamentally connected to redox signaling, cellular communication, and acclimation. The thiol-disulfide network is central part of the stress sensing and processing response and integrates information input with redox regulation.

Recent Advances: Prxs function both as redox sensory system within the network and redox-dependent interactors. The processes directly or indirectly targeted by Prxs include gene expression, post-transcriptional reactions, including translation, post-translational regulation, and switching or tuning of metabolic pathways, and other cell activities. The most advanced knowledge is available for the chloroplast 2-CysPrx wherein recently a solid interactome has been defined. An in silico analysis of protein structure and coexpression reinforces new insights into the 2-CysPrx functionality.

Critical Issues: Up to now, Prxs often have been investigated for local properties of enzyme activity. In vitro and ex vivo work with mutants will reveal the ability of Prxs to interfere with multiple cellular components, including crosstalk with $\mathrm{Ca}^{2+}$-linked signaling pathways, hormone signaling, and protein homeostasis.

Future Directions: Complementation of the Prxs knockout lines with variants that mimic specific states, namely devoid of peroxidase activity, lacking the oligomerization ability, resembling the hyperoxidized decamer, or with truncated C-terminus, should allow dissecting the roles as thiol peroxidase, oxidant, interaction partner, and chaperone. Antioxid. Redox Signal. 28, 609-624.
\end{abstract}

Keywords: thiol peroxidase, signaling, post-translational modifications, redox network, 2-cysteine peroxiredoxin, interactome, chloroplast

\section{Biochemical Features and Dynamics of Peroxiredoxins}

$\mathbf{T}$ HE CENTRAL CHEMICAL GROUP in peroxiredoxins (Prxs) is the cysteinyl thiol with low $\mathrm{p} K$ value, the peroxidatic Cys. Together with plant glutathione peroxidases that in animals and Chlamydomonas function with a selenocysteine instead of the catalytic cysteine found in plants, Prxs constitute the thiol peroxidases of plant cells $(23,32)$. The thiolbased mechanism is independent of cofactors such as metal ions or prosthetic groups. Thus thiol peroxidases employ a simple nucleophilic attack mechanism in the primary catalytic reaction $(46,100)$.

Circumstantial evidence indicates that Prxs are ancient high-affinity enzymes for peroxide detoxification when atmospheric oxygen still was very low (31). After formation of the sulfenic acid derivative by reaction of $\mathrm{Cys}_{\mathrm{P}}$ with the peroxide substrate, the $\mathrm{Cys}_{\mathrm{P}}$ thiol is subjected to various redox modifications ranging from disulfide, sulfinic to sulfonic acid derivatives. The site of the resolving cysteine $\left(\mathrm{Cys}_{\mathrm{R}}\right)$ and the quaternary interaction interfaces define the distinct forms

Department of Biochemistry and Physiology of Plants, Faculty of Biology, University of Bielefeld, Bielefeld, Germany.

(C) Michael Liebthal, et al., 2018; Published by Mary Ann Liebert, Inc. This article is available under the Creative Commons License CC-BY-NC (http://creativecommons.org/licenses/by-nc/4.0). This license permits non-commercial use, distribution and reproduction in any medium, provided the original work is properly cited. Permission only needs to be obtained for commercial use and can be done via RightsLink. 
of Prxs that have emerged during evolution and exhibit differential specificities toward peroxide substrates, distinct subcellular localization, and regulatory capabilities. The often highly abundant Prxs stabilize redox homeostasis, transmit redox information, and control cell metabolism, in particular, photosynthesis.

Recent research focused on the spatial and temporal occurrence of post-translational modifications (PTMs) of Prxs and their mechanistic coupling to redox reaction chains. Oxidations on thiols occur with increasing oxidation number starting with disulfide $(\mathrm{S}-\mathrm{S})$, sulfenic $(-\mathrm{SOH})$, sulfinic $\left(-\mathrm{SO}_{2} \mathrm{H}\right)$ to sulfonic $\left(-\mathrm{SO}_{3} \mathrm{H}\right)$ acid. Except for the sulfonic acid, each of these modifications bears functional significance. Reduction is needed for regeneration $(26,106)$.

Disulfide formation in 2-CysPrx causes a major conformational switch because the $\mathrm{C}$-terminus carrying the $\mathrm{Cys}_{\mathrm{R}}$ residue has to locally unfold and move to the sulfenylated peroxidatic cysteine. This mechanism destabilizes the dimer-dimer interface resulting in loss of decamer integrity (121). This effect can be analyzed by size exclusion chromatography (SEC) wherein disulfide-bridged oxidized Arabidopsis 2-CysPrx separates as dimer, whereas the reduced form separates as decamer and dimer (62). The oxidized dimers may ease rereduction by NADPH- dependent thioredoxin reductase $\mathrm{C}$ (NTRC) or thioredoxins (Trxs) such as Trx-x, CDSP, or Trx-m $(26,96)$.

Hyperoxidation of the Cysp to sulfinic acid in the $\mathrm{H}_{2} \mathrm{O}_{2}$ detoxification cycle occurs with a certain frequency of about 1 out of 250 turnovers for Arabidopsis thaliana 2-CysPrx when exposed to a constant $\mathrm{H}_{2} \mathrm{O}_{2}$ generation rate of $1 \mu M \mathrm{H}_{2} \mathrm{O}_{2} \min ^{-1}$ from a glucose oxidase system (62). Hyperoxidation opens up additional possibilities for signaling because the hyperoxidized decamer adopts a more stable conformation than the reduced decamer and at least in vitro acts as chaperone preventing precipitation of citrate synthase and insulin at high temperature and reducing conditions, respectively (59). Moreover, $S$-nitrosylation (36, 104), phosphorylation (see Table 2), Cys-glutathionylation (84), and Lys-acetylation (83) are reported PTMs of Prxs, which, in turn, alter Prx properties. Thus activity and function of Prxs are integrated into multiple signaling pathways as discussed hereunder.

Basically, there exist two common but in fact highly related classifications of Prxs. Prxs are assigned to six distinct groups based on their amino acid sequence, cysteine localization, interaction interface, and N-/C-terminal extensions. Classification and naming of Prxs changed along with novel discoveries, improved analytical methods, and also

Table 1. Overview of Prx Subfamilies in Plants, Mammals, and Yeast

\begin{tabular}{|c|c|c|c|c|c|c|c|}
\hline \multirow[b]{2}{*}{ Type } & \multicolumn{3}{|c|}{ Plant } & \multicolumn{3}{|c|}{ Mammal } & \multirow{2}{*}{$\begin{array}{c}\text { Yeast } \\
\text { Saccharomyces }^{\text {cerevisiae }^{(1)}}\end{array}$} \\
\hline & $\begin{array}{l}\text { Arabidopsis }_{\text {thaliana }^{(1)}}\end{array}$ & $\begin{array}{l}\text { Oryza }^{(1)} \\
\text { sativa }^{(1)}\end{array}$ & $\begin{array}{c}\text { Beta } \\
\text { vulgaris }^{(2)}\end{array}$ & $\begin{array}{c}\text { Homo }^{(1)} \\
\text { sapiens }^{(1)}\end{array}$ & $\begin{array}{c}\text { Mus } \\
\text { musculus }^{(1)}\end{array}$ & 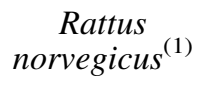 & \\
\hline A & $\begin{array}{l}\text { 2-CysPrxA (cl) } \\
\text { Q96291 } \\
\text { 2-CysPrxB (cl) } \\
\text { Q9C5R8 }\end{array}$ & $\begin{array}{c}\text { 2-CysPrxA (cl) } \\
\text { Q6ER94 } \\
-\end{array}$ & $\begin{array}{l}\text { 2-CysPrx (cl) } \\
\text { XM_010685313.1 }\end{array}$ & $\begin{array}{c}\text { Prdx }(c t) \\
\text { Q06830 } \\
\text { Prdx2(ct) } \\
\text { P32119 } \\
\text { Prdx3(mt) } \\
\text { P30048 } \\
\text { Prdx4(ct) } \\
\text { Q13162 }\end{array}$ & $\begin{array}{c}\text { Prdx1(ct) } \\
\text { P35700 } \\
\text { Prdx2(ct) } \\
\text { Q61171 } \\
\text { Prdx3(mt*) } \\
\text { P20108 } \\
\text { Prdx4(ct*) } \\
\text { O08807 }\end{array}$ & $\begin{array}{c}\text { Prdx1(ct) } \\
\text { Q63716 } \\
\text { Prdx2(ct) } \\
\text { P35704 } \\
\text { Prdx3(mt*) } \\
\text { Q9Z0 V6 } \\
\text { Prdx4 (ct*) } \\
\text { Q9Z0 V5 }\end{array}$ & $\begin{array}{l}\text { Prx1, Tsa1 } \\
\text { (ct) P34760 } \\
\text { Prx2, Tsa2 } \\
\text { (ct) Q04120 }\end{array}$ \\
\hline B & $\begin{array}{l}\text { 1-CysPrx } \\
\text { (ct, n) } \\
\text { O04005 }\end{array}$ & $\begin{array}{l}\text { 1-CysPrx } \\
\left(\mathrm{ct}, \mathrm{n}^{*}\right) \\
\text { P0C5C9 }\end{array}$ & $\begin{array}{l}\text { 1-CysPrx (ct, n) } \\
\text { XM_010669313.1 }\end{array}$ & $\begin{array}{l}\text { Prdx6(ct) } \\
\text { P30041 }\end{array}$ & $\begin{array}{l}\text { Prdx6(ct) } \\
\text { O08709 }\end{array}$ & $\begin{array}{l}\text { Prdx6(ct) } \\
\text { O35244 }\end{array}$ & $\begin{array}{l}\mathrm{mTpx}(\mathrm{mt}) \\
\text { P34227 }\end{array}$ \\
\hline $\mathrm{C}$ & $\begin{array}{l}\text { PrxQ }(\mathrm{cl}) \\
\text { Q9LU86 }\end{array}$ & $\begin{array}{l}\text { PrxQ (cl) } \\
\text { P0C5D5 }\end{array}$ & $\begin{array}{l}\text { PrxQ (cl) } \\
\quad \text { XM_010689254.1 }\end{array}$ & - & - & - & $\begin{array}{l}\text { nTpx (n) } \\
\text { P40553 }\end{array}$ \\
\hline $\mathrm{D}$ & $\begin{array}{l}\text { PrxIIA }(-) \\
\text { Q7G959 }\end{array}$ & - & - & $\begin{array}{l}\text { Prdx5(ct,mt,pr) } \\
\quad \text { P30044 }\end{array}$ & $\begin{array}{l}\text { Prdx5 } \\
\text { (ct,mt,pr) } \\
\text { P99029 }\end{array}$ & $\begin{array}{l}\text { Prdx5 } \\
\text { (ct,mt,pr) } \\
\text { Q9R063 }\end{array}$ & $\begin{array}{c}\text { cTpx3 (ct) } \\
\text { P38013 }\end{array}$ \\
\hline & $\begin{array}{l}\text { PrxIIB }(\mathrm{ct}) \\
\text { Q9XEX2 }\end{array}$ & $\begin{array}{r}\text { PrxIIB (ct) } \\
\text { Q9FR35 }\end{array}$ & $\begin{array}{l}\text { PrXIIB (ct) } \\
\text { XM } 010693075.1\end{array}$ & & & & \\
\hline & $\begin{array}{l}\text { PrxIIC (ct) } \\
\text { Q9SRZ4 } \\
\text { PrxIID (ct) } \\
\text { O222711 }\end{array}$ & - & - & & & & \\
\hline & $\begin{array}{l}\text { PrxIIE (cl) } \\
\text { Q949 U7 } \\
\text { PrxIIF (mt) } \\
\text { Q9 M7T0 }\end{array}$ & $\begin{array}{c}\text { PrxIIE1 (cl*) } \\
\text { Q69TY4 } \\
\text { PrxIIE2 (cl*) } \\
\text { Q7F8S5 } \\
\text { PrxIIF (mt*) } \\
\text { Q9SDD6 }\end{array}$ & $\begin{array}{l}\text { PrxIIE (cl) } \\
\text { XM_010685588.1 } \\
-\end{array}$ & & & & \\
\hline
\end{tabular}

Based on Hofmann et al. (49), Prxs were sorted with regard to their respective biochemical property of peroxidative cysteines and structure. In addition to the two plant model organisms, the newly sequenced genome of Beta vulgaris was included as additional example (51). For further information concerning subcellular localization, function, biochemical properties, and crystallographic data of annotated proteins, see the respective Uniprot entry (1) or reference sequence for B. vulgaris (2). Subcellular localization is given in parentheses as follows: nucleus $=\mathrm{n}$, cytosol $=\mathrm{ct}$, chloroplast $=\mathrm{cl}$, mitochondria $=\mathrm{mt}$, endoplasmatic reticulum $=$ er, peroxisome $=$ pr, not known $=-$. An * indicates manual assertion inferred from sequence similarity. 
depending on the organism. Table 1 provides an overview on the Prx nomenclature. The basic classification follows that of (49). Additional information was retrieved from the Prx classification index (PREX) and concerned sequence and structural features (111). Types A, B, C, and D Prxs are present in plants, whereas types $\mathrm{E}$ and $\mathrm{F}$ are only found in some bacteria. Prxs are often classified in typical 2-cysteine (type A), typical 1-cysteine (type B), and atypical 2-cysteine Prxs, which cover most Prxs. The latter are separated into PrxQ (type C) and type II Prx (type D). Additional features distinguishing Prx types concern the regeneration and subunit composition and interaction mechanisms. The assignment considers the position of the peroxidatic nucleophilic cysteine $\left(\mathrm{Cys}_{\mathrm{P}}\right)$ and the presence, position, or absence of a $\mathrm{Cys}_{\mathrm{R}}$ capable of reacting with oxidized $\mathrm{Cys}_{\mathrm{P}}$ (sulfenic acid). The minimal unit can be monomer or dimer. In type A Prx, dimers form intermolecular disulfide bridges $\left(+\mathrm{Cys}_{\mathrm{R}}\right)$. Dimers of type B Prx lack the $\mathrm{Cys}_{\mathrm{R}}$ and are linked noncovalently. Type $\mathrm{C}$ monomers carry a $\mathrm{Cys}_{\mathrm{R}}$ and generate intramolecular disulfide bonds. Type D Prxs form intramolecular disulfide bonds with $\mathrm{Cys}_{\mathrm{R}}$ as well and noncovalently linked dimers. Trxs, NTRC, glutaredoxin (Grx)/glutathione (GSH), or other electron donors mentioned in the text restore the catalytically active $\mathrm{Cys}_{\mathrm{P}}$ in Prxs belonging to types A, C, and D. In the $\mathrm{Cys}_{\mathrm{R}^{-}}$ free type B Prx, the sulfenic acid immediately reacts with a thiol or another reductant such as ascorbate. The review by Dietz (31) covers many aspects of the Prx family in plants. A genome-wide search allowed us to define the minimum set of six Prxs expressed in all higher plants [31], namely one 1-CysPrx related to embryogenesis, one chloroplast PrxQ, one plastid 2-CysPrx, and one each of cytosolic, mitochondrial, and plastidic type II Prxs. Variation of this minimum set of Prxs frequently occurs with multiple isoforms such as two 2-CysPrxs and three cytosolic PrxIIB-D in A. thaliana, but two Prx II in poplar (PrxIIB, PrxIIC) and only one in rice (PrxIIB).

All Prxs and glutathione peroxidases (Gpxs) have a highly conserved structure based on the typical Trx-like fold. Based on this observation, the hypothesis emerged that all Prxs have a common Trx ancestor. The proper Trx-like fold is built from a $\beta \alpha \beta \beta \beta \alpha$ structure (97). The expanded structure of Prxs consists of seven-stranded $\beta$-sheets surrounded by five $\alpha$ helices in $\beta_{1}-\beta_{2}-\alpha_{1}-\beta_{3}-\alpha_{2}-\beta_{4}-\alpha_{3}-\beta_{5}-\beta_{6}-\alpha_{4}-\beta_{7}-\alpha_{5}$ topology. The properties of the catalytic center composed of the Cys $\mathrm{s}_{\mathrm{P}}$ in its conserved environment were analyzed by crystallography, bioinformatics, and activity assays. These amino acids are arranged in a reaction pocket between sheets $\beta_{3}$ and $\beta_{6}$, helix $\alpha_{2}$, and the loop connecting $\alpha_{2}$ and $\beta_{3}$ (81). This position is conserved whereas the resolving Cys is variably positioned in helices $\alpha_{2}, \alpha_{3}$, and $\alpha_{5}$ or in the C-terminal region for intermolecular resolving reaction (91). The physicochemical properties of the specified amino acid residues combined with a nucleophilic displacement reaction $\left(S_{N} 2\right)$ provide a framework for the enzymatic mechanism (Fig. 1). The Arg and Thr/Ser together with the Cys thiolate support the binding of peroxides. The oxygen-thiolate interaction polarizes the terminal oxygen to facilitate the $\mathrm{Cys}-\mathrm{S}-\mathrm{O}$ bond formation. The hydrophobic Pro protects the highly active cysteine site from exposure to the solvent and promotes the high peroxide specificity. Amino acid sequence comparison between At2-CysPrx, PsPrxIIF, and BtPrx3 underlines a conserved positioning for flanking amino acids and peroxidative cysteine in different Prxs and across species such as BtPrx3
A
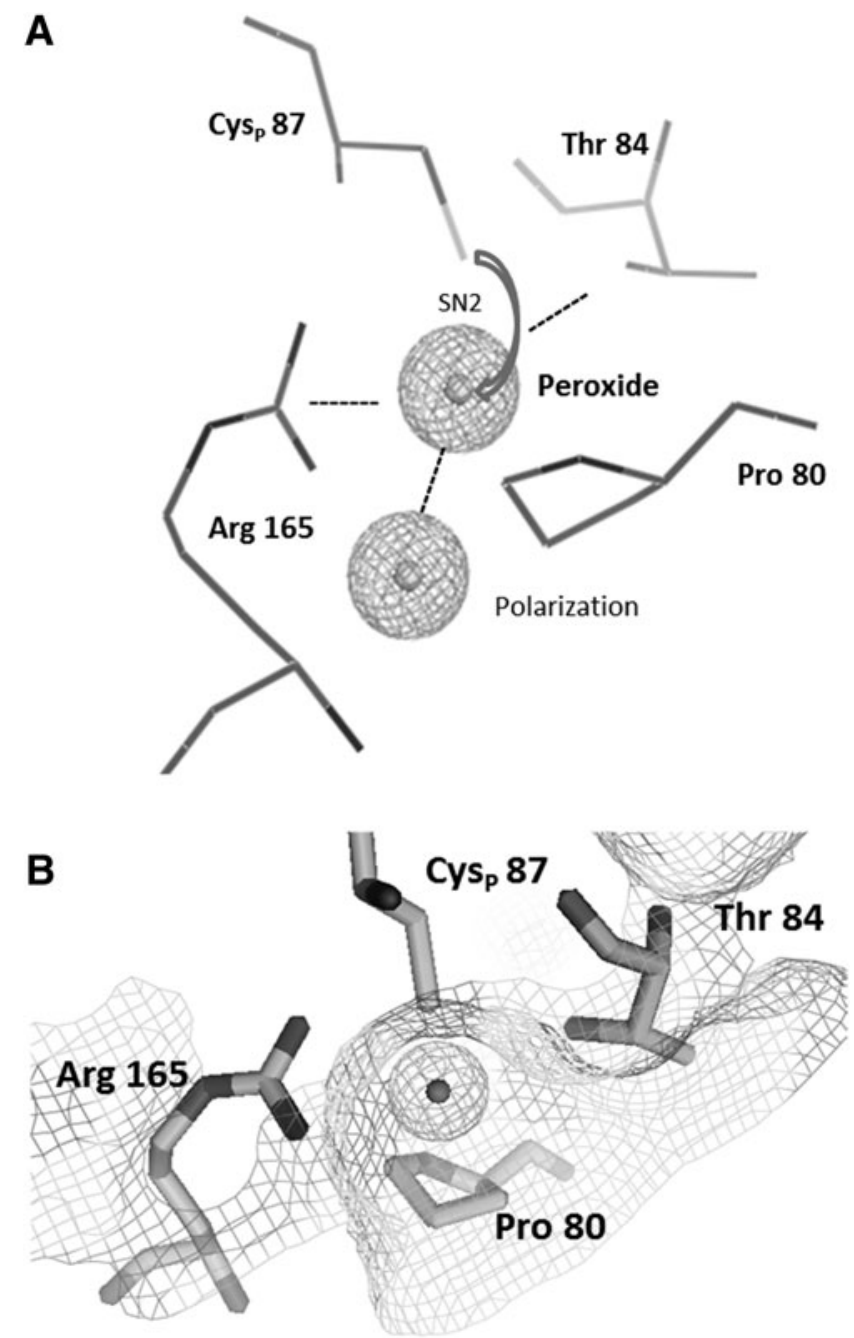

FIG. 1. Peroxide binding pocket with conserved amino acids. (A) Graphical presentation of the catalytic center of the mitochondrial PrxIIF of pea. The template was accessed from RCSB PDB with the model of 2PWJ (2007) at $2.8 \AA$ resolution. The conserved amino acids $\mathrm{Cys}_{\mathrm{P}}$ 87, $\mathrm{Arg} 165$, Thr 84, and Pro 80 are depicted using PyMOL (29). The peroxyl moiety was placed in appropriate position in the catalytic reaction pocket to simulate peroxide binding. (B) Potential surface mesh is laid on the active site residues and the terminal oxygen of substrate.

102PXXXTXXCX...XR185, PsPrXIIF 80PXXXTXXCX... XR165, and At2-CysPrx 162PXXXTXXCX...XR195. Around $83 \%$ of all $\mathrm{Cys}_{\mathrm{P}}$ display a $\mathrm{p} K_{\mathrm{a}}$ between 5.1 and 6.3 and thus are present in the thiolate form $\left(\mathrm{Cys}^{-} \mathrm{S}^{-}\right)$at physiological pH of, for example, 7.2 in the cytosol (93). The nucleophilic characteristics of the catalytic center originating from deprotonation at low- $\mathrm{pH}$ value (low $\mathrm{p} K_{\mathrm{a}}$ ) generates a rather efficient peroxide detoxification microenvironment with high substrate affinity in the low micromolar range (39, 93). However, the turnover number is low with 10-250 reactions/min. The catalytic efficiency of Prxs is in the range of $10^{5}-10^{8} M^{-1} \mathrm{~s}^{-1}$ and depends strongly on the thiol regeneration mechanism (96). The variation by three orders of magnitudes points to diversification of Prxs across all kingdoms. As shown for AhpC in Salmonella (86) and Prdx2 in human systems (66), an efficiency of $10^{7} M^{-1} \mathrm{~s}^{-1}$ for $\mathrm{H}_{2} \mathrm{O}_{2}$ is 


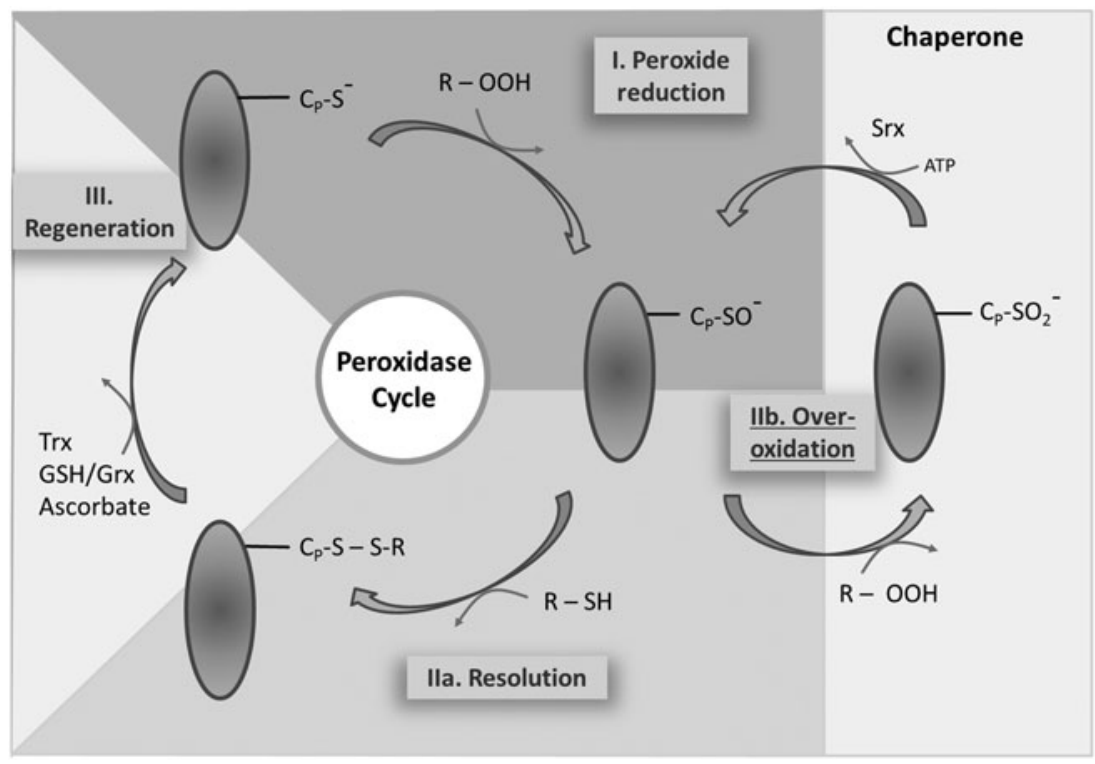

FIG. 2. Schematics of the peroxidase cycle and hyperoxidation cycle of Prxs. (I) Reduced Prx scavenges peroxides $\left(\mathrm{H}_{2} \mathrm{O}_{2}\right)$ releasing water. The peroxidatic cysteine $\left(\mathrm{Cys}_{\mathrm{P}}\right)$ forms a sulfenic acid derivative. (IIa) In the resolving step, a second cysteine $\left(\mathrm{Cys}_{\mathrm{R}}\right)$ attacks the sulfenic acid to form an inter- or intramolecular disulfide. (IIb) The reaction with an additional peroxide may hyperoxidize $\mathrm{CyS}_{\mathrm{P}}$ to the sulfinic acid form. Hyperoxidation of 2-CysPrx can be retroreduced by the ATPdependent sulfiredoxin, returning sulfenic forms into the cycle. (III) The regeneration step is catalyzed by a thiol reductantlike NTRC or Trx, reducing the catalytic and resolving cysteine and converting the Prxs to an active thiol peroxidase.

similar to catalase $\left(10^{7} M^{-1} \mathrm{~s}^{-1}\right)$ or selenocysteine-based Gpx $\left(10^{8} M^{-1} \mathrm{~s}^{-1}\right)(122)$.

The thiol peroxidase cycle consists of three steps: the reduction of the peroxide substrate and generation of the sulfenic acid form of $\mathrm{Cys}_{\mathrm{P}}$, the resolution of the sulfenic acid to release water, and the regeneration of the thiol by electron transmitters (Fig. 2). (I) After binding of the peroxide to $\mathrm{Cys}_{\mathrm{P}}$, the oxygen-oxygen bond of the peroxide is disrupted and the corresponding alcohol from alkylhydroperoxide, water from $\mathrm{H}_{2} \mathrm{O}_{2}$, and nitrite from peroxinitrite are released. The active thiol group of the $\mathrm{Cys}_{\mathrm{P}}$ is converted to a sulfenic acid (-SOH) group. (IIa) The $\mathrm{Cys}_{\mathrm{R}}$ on the same or a different molecule attacks the sulfenic acid and forms an interor intramolecular disulfide bond (Cys-S-S-Cys). The disulfide bonded Prx adopts the inactive form. (IIb) Peroxide competes with the $\mathrm{Cys}_{\mathrm{R}}$ for the sulfenic acid group. A second oxidation can take place before the resolving step occurs. This is considered as a regulatory mechanism that allows for inactivation and local peroxide accumulation in line with the flood gate model. The sulfinic acid is described as hyperoxidized form of Prxs. Its conversion to the active thiol peroxidase requires an additional regeneration step by sulfiredoxin (Srx) (94). (III) In the final step, the disulfide is reduced by specific redox transmitters such as Trxs, NTRC, Grx in combination with GSH, and ascorbate (reviewed in Ref. 31).

\section{Prx Properties of Typical 1-CysPrx and 2-CysPrx}

\section{1-cysteine peroxiredoxin}

Typical 1-cysteine peroxiredoxins (1-CysPrxs) belong to the B-type class and have a homodimer interface like Atype Prxs. 1-CysPrxs carry the $\mathrm{Cys}_{\mathrm{P}}$ but lack the $\mathrm{Cys}_{\mathrm{R}}$. The sulfenic acid is resolved by other interaction partners. Information on plant 1-CysPrxs is still scarce. They are pref- erentially expressed in seeds wherein, for example, in barley they are found in the nucleus of aleurone and scutellum cells (113). Owing to its mostly confined expression in seeds, 1CysPrx has mostly moved out of focus of current redox research. In 2009, Pulido and coworkers described the NTR/ Trx system as electron donor for regeneration of 1-CysPrx (95). Enzyme assays with heterologously expressed wheat 1CysPrx showed NTR-dependent peroxidase activity as well as localization in the nucleus as shown by immunolabeling. With $70 \mathrm{kDa}$, the molecular mass of NTR dimers is too big to diffuse through nuclear pores and thus the translocation mechanism to the nucleus remains elusive in the light of the missing nuclear localization sequence (95). It may be speculated that 1-CysPrx is taken piggyback by other cargo. Oxidized 1-CysPrx can be regenerated with ascorbate as electron donor. The efficiency appears to be low compared with that with the Trx system both for yeast mTpx and rat Prdx6. The antioxidant role of erythroascorbate remains a matter of debate despite the fact that the $\mathrm{k}_{\mathrm{cat}} / \mathrm{K}_{\mathrm{M}}$ values for erythroascorbate with $10^{4} M^{-1} \mathrm{~s}^{-1}$ were one order of magnitude below that of Trx with $10^{5} M^{-1} \mathrm{~s}^{-1}$ (72). A recent report compared kinetic reactivity of the 1-CysPrx sulfenic acid with ascorbate across many species (3). The catalytic efficiencies $\mathrm{k}_{\text {cat }} / \mathrm{K}_{\mathrm{M}}$ for $\mathrm{H}_{2} \mathrm{O}_{2}$ decomposition were similar for yeast, bacteria, plant, and human 1-CysPrx with values of around $10^{3} M^{-1} \mathrm{~s}^{-1}$. The significance of the ascorbatedependent regeneration in plants is still difficult to judge. Its contribution likely depends on the relative concentration of the various reductants, because in addition to Trx also GSH and glutathione- $S$-transferase and glutaredoxin, respectively (GST/GSH, Grx/GSH), regenerate 1-CysPrx at least in mammalia (64) and yeast (87). The link of 1-CysPrx to various regeneration systems may increase the viability of seeds facing harsh environmental conditions. Indeed Arabidopsis 
seeds overexpressing the PER1 homologue of Nelumbo nucifera (24) germinated better by $25 \%-50 \%$ than wild type (WT) in controlled deterioration treatments. The seedlings accumulated fivefold more biomass when grown on $\mathrm{ABA}$ for 10 days. These observations and increased germination at $50^{\circ} \mathrm{C}$ underpin the crucial role of 1-CysPrx in the redox network of seeds.

\section{2-cysteine peroxiredoxin}

Typical 2-CysPrxs interact through an obligate monomermonomer interface orientated in parallel to the $\beta$-sheet bundles (B-type interface) in the Trx fold. In addition, intermolecular thiol-disulfide bonds are formed in the homodimer (46). 2-CysPrx is a highly abundant protein in the chloroplast stroma (100-120 $\mu M$ in total for both 2-CysPrx A and $\mathrm{B}$ in $A$. thaliana) and likely involved in redox regulation in both the stroma and near the thylakoids $(58,89)$. Immunolabeling of 2-CysPrx with gold nanoparticles-conjugated antibodies revealed a location vicinal to thylakoid membranes of barley chloroplasts for $72 \%$ of all counted particles. Control analyses of etioplasts resulted in less (32\%) label in proximity to the prolammelar body. Preimmune serum gave no such label in chloroplasts (58). Muthuramalingam et al. (77) detected 2CysPrx in isolated photosystem II-enriched particles. Cerveau et al. (21) analyzed carefully washed membranes obtained from control or water-stressed A. thaliana WT and srx mutants and failed to detect 2-CysPrx in the thylakoid fraction. These controversial observations indicate that 2-CysPrx possibly associates with the thylakoids in a dynamic and conformational state-dependent manner and that this association is lost during extensive washing.

2-CysPrx adopts five different quaternary conformations. The reduced dimer, the reduced decamer, and the oxidized dimer take part in the peroxidase cycle already described. The hyperoxidized decamer and the hyperoxidized hyperaggregate realize the chaperone function of 2-CysPrx (60). Site-directed mutated variants of Arabidopsis 2-CysPrx revealed variable chaperone activity with lowest activity of a C-terminally truncated version and a disulfide-bond mim- icking variant and highest activity for the hyperoxidationstate-mimicking variant C54D (59). 2-CysPrx, together with PrxIIE and PrxQ, is part of the complex thiol-disulfide network of the chloroplast, functions as redox sensors, and participates in regulation and maintenance of metabolic homeostasis (Fig. 3) (30). Owing to its high affinity and reactivity, 2-CysPrx may be considered as primary reactive oxygen species (ROS) sensor. In line with this hypothesis, 2CysPrx was among the most sensitive targets of oxidation by $\mathrm{H}_{2} \mathrm{O}_{2}$ in vitro and methylviologen treatment in vivo (76). Regeneration is achieved by, for example, NTRC and Trx-x, but also by Trx-f1 and thereby is connected to the electron input elements ferredoxin (Fd) and NADPH $(26,90)$. The peroxidase activity is limited by the slow regeneration reaction. Increasing ROS turnover may disturb this process by hyperoxidizing the catalytic cysteines after $\sim 250$ peroxidase cycles $(56,62)$. Then the alternative ATP-dependent regeneration system of Srx regenerates the active 2-CysPrx in a very slow reaction with about $1 \mathrm{~min}^{-1}$. Initially, Srx was thought to be specific for 2-CysPrx in the chloroplast until Iglesias-Baena et al. (53) proved the dual targeting of Srx also to the mitochondrium. The data revealed that Srx reduces hyperoxidized PrxIIF in the mitochondrion.

The occurrence and functional link between oligomerization, hyperoxidation, and chaperoning still need to be further investigated for structural and functional features. Noichri and coworkers (82) suggested that stacked reduced decamers act as precursors for stacked hyperoxidized aggregates in Saccharomyces cerevisiae. Two different high molecular weight (HMW) fractions were identified by SEC and native polyacrylaminde gel electrophoresis (PAGE) or SDS-PAGE, depending on the redox state. The occurrence of hyperoxidized 2-CysPrx follows a circadian rhythm in eukaryotic cells. The circadian hyperoxidation does not depend on transcriptional circuits and correlates with release of $\mathrm{H}_{2} \mathrm{O}_{2}$ from mitochondria in yeast (101). It should be noted that the fraction of hyperoxidation is rather small with an estimated share of up to $1.6 \%$ of total 2-CysPrx in erythrocytes of WT mice (25). The circadian oscillation of 2-CysPrx hyperoxidation
FIG. 3. Role of Prxs in the thioldependent redox network. Environmental fluctuations and metabolic disequilibria generate ROS that drain electrons from Prxs that may be considered as redox sensors. Oxidized thiol peroxidases such as Prxs are reduced by redox transmitters, which receive electrons from redox input elements such as NADPH and ferredoxin (Fd). The redox transmitters (Trx, Grx, NTRC, and ACHT) control the redox state of redox targets that act as thiol switches, regulate virtually all cellular processes, and modulate signaling that feeds back to the reducing site and the generator systems. Prxs and ROS may also directly oxidize target proteins by proximity-based oxidation. NTRC, NADPH-dependent thioredoxin reductase $\mathrm{C}$; ROS, reactive oxygen species.

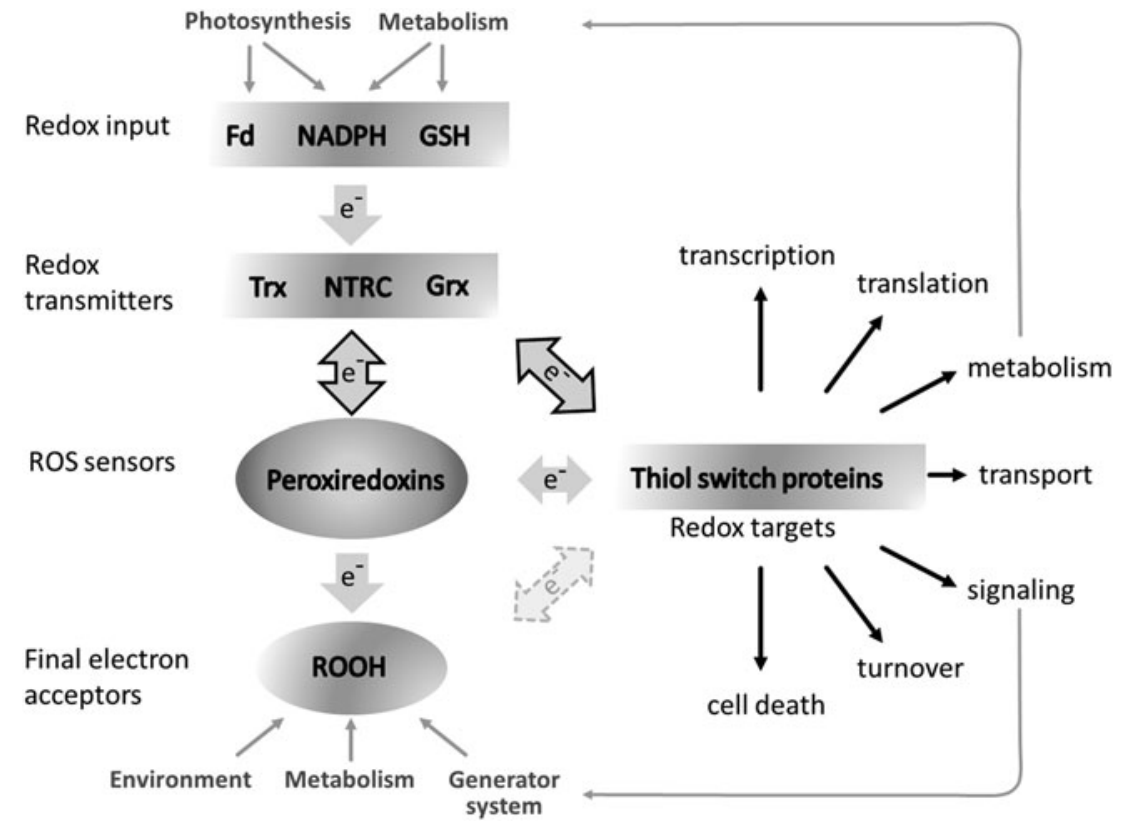


also occurs in A. thaliana and other plants (34). But again the amount subjected to hyperoxidation is low (21). For the time being, the circadian appearance of hyperoxidized 2-CysPrx is considered as indicator for daily alterations in cell redox milieu possibly linked to specific metabolic activities such as FeS center assembly. A major accumulation of hyperoxidized 2CysPrx was also not seen under temperature or water stress (21). The absence of HMW complexes was interpreted as indicator that the chaperone function might be less important than previously proposed. In line with this suggestion, hyperoxidized 2CysPrx could neither be detected in nonreducing gels nor in Western blots using sulfinic/sulfonic antibody. Srx and NTRC transcript levels coding for the main regulators of 2-CysPrx redox state were unaffected under stress (94).

Apparently the system consisting of 2-CysPrxs, NTRC, and Srx guarantees a rather stable redox state of 2-CysPrx under most conditions. Interestingly, hyperoxidized 2CysPrx was found in plants lacking NTRC. 2-CysPrx can turn hyperoxidized only if the peroxidase cycle is running. Therefore, the hyperoxidation in $n$ trc background proves that 2-CysPrx receives sufficient electrons by NTRC-independent components such as Trx-x, CDSP32, Trx-f, and Trx-m to sustain the peroxidase cycle. While questioning the significance of circadian oscillations in hyperoxidation, the authors suggest a light-dependent regulation of 2-CysPrx hyperoxidation dependent on photosynthetic electron transport (PET) and the Fd/FTR/Trx system (21). Alternatively, the small fraction of hyperoxidized 2-CysPrx might realize a gain of function by specific interaction with targets apart from general chaperoning.

A peculiar feature of reduced 2-CysPrx is its dynamic transition from dimer to decamer. Several physicochemical parameters influence this transition and the stability of the reduced decamer, in particular $\mathrm{pH}$, ionic strength, redox potential, and its own concentration $(12,62,75)$ (Fig. 4A). Below the critical transition concentration (CTC), reduced 2-
CysPrx exclusively adopts the dimeric conformation. With extremely high cooperativity, reduced dimers assemble to decamers above the CTC similar to the abrupt transition of free lipids to lipid micelles above the critical micelle concentration. SEC, isothermal titration microcalorimetry (ITC), and dynamic light scattering defined the CTC with about $2.2 \mu M$ at $\mathrm{pH} 8$ and $4.2 \mu M$ at $\mathrm{pH}$ 7.2. The $\mathrm{pH}$ dependency might possibly be linked to a histidine in the dimer-dimer interface, which is protonated at lower $\mathrm{pH}$. His protonation may decrease the ability to form decamers.

Stromal proteins interfere with the CTC, for example, the cyclophilin 20-3 (Cyp20-3), a peptidyl-prolyl-cis/trans isomerase that regulates cysteine synthesis (85). In contrast, titration of 2-CysPrx in $200 \mu \mathrm{g} / \mathrm{ml}$ stromal protein extract significantly decreased the CTC to $0.6 \mu M$ (62). Apparently complex and dynamic mechanisms regulate the dimer-decamer equilibrium, wherein a relatively small portion of dimers interact with several partners, or the binding partners of the decamer affect the equilibrium between dimer and decamer. The schematic depicted in Figure 4B summarizes the conformational states and their proposed or proven functions.

\section{The Prx Interactome}

2-CysPrxs, such as PrxIIE and PrxQ, are part of the chloroplast redox-regulatory network and are targeted by ROS as oxidants and electron transmitters as reductants (Fig. 3). Chloroplast Prxs and Gpx are preferentially reduced by some members of the whole chloroplast Trx family, consisting of Trx-f1, -f2, m1-4, -x, -y1, -y2,-z, 9 Trx-like proteins (NTRC, CDSP32, ACHT 1-4 [atypical cysteine histidine-rich Trx], and APR-1-3 [protein disulfide isomerase-like proteins]). The thiol peroxidases and two Asc-dependent peroxidases (thylakoid Apx and stromal Apx) reveal distinct suborganellar localization in the plastids $(14,61,71)$.
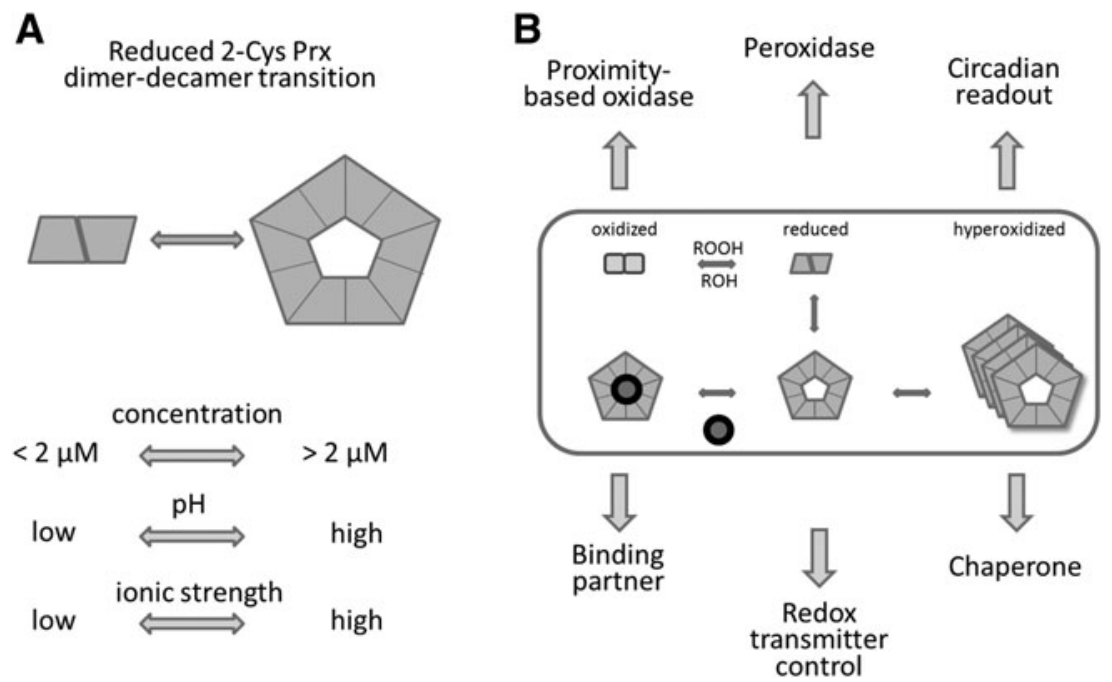

FIG. 4. Multifunctionality of 2-CysPrx in plants. (A) Reduced 2-CysPrx adopts a dimer-decamer equilibrium. Several physicochemical parameters affect this equilibrium: the 2-CysPrx concentration, the $\mathrm{pH}$, and the ionic strength. The critical transition concentration defines the minimum concentration for effective decamer association. Low $\mathrm{pH}$ and low ionic strength favor the dimer, whereas high $\mathrm{pH}$ and ionic strength support oligomerization. (B) 2-CysPrx is involved in multiple cellular processes based on its redox-dependent conformational state, namely as thiol peroxidase, oxidant of redox transmitters in the redox network, proximity-based oxidase, chaperone, protein interactor, and also as redox indicator in the circadian rhythm. 
The stromal 2-CysPrx is part of an intricate protein-protein interaction network. NTRC and Trx-x, together with CDSP32, are described as the predominant reductants of oxidized 2CysPrx in vitro and in vivo and regenerate the active thiol peroxidase (Fig. 2) (14, 17). Reduced Cyp20-3 interacts with reduced 2-CysPrx as shown by fluorescence resonance energy transfer in protoplasts, ITC, and in vitro binding assays $(62,77)$.

Cyp20-3 binds 12-oxophytodienoic acid (OPDA), the precursor of jasmonates, with high affinity $\left(\mathrm{K}_{\mathrm{d}}=196 \mathrm{nM}\right)(85)$. OPDA-liganded Cyp20-3 activates the chloroplast cysteine synthase complex consisting of serine acetyltransferase 2.1 (Serat) and O-acetylserine thiol lyase-B (OASTL-B). However, Cyp20-3 may not be necessary for chloroplast cysteine synthesis because formation of the cysteine synthase complexes occurs spontaneously $\left(\Delta \mathrm{G}=-33 \mathrm{kcal} \mathrm{mol}^{-1}\right)(112)$. In addition, only about $20 \%$ of cellular GSH synthesis derives from chloroplast Serat 2.1/OASTL-B, whereas $80 \%$ originate from mitochondrial and cytosolic cysteine synthase (112). Nonetheless, Cyp20-3 is regarded as a mediator of stressdependent redox responses. The interaction between 2-CysPrx and Cyp20-3 occurs under reducing conditions and is suggested to compete with the activation of the cysteine synthase depending on the binding of OPDA and a potential conformational shift. This mechanism would allow for a negative regulatory feedback, slowing down stress-induced cysteine synthesis after re-establishing redox homeostasis.

Dangoor et al. (27) described an interaction of 2-CysPrx with ACHT1 as part of the chloroplast redox-regulatory network. 2-CysPrx is regarded as oxidant of ACHT1, which is suggested to regulate metabolism and acclimation processes, for example, during light-dark transitions of photosynthesis. Furthermore, ACHT4 also interacts with 2-CysPrx using a similar mechanism like ACHT1. The ADP-glucose pyrophosphorylase is an essential enzyme of starch synthesis. Its small subunit is implicated in the redox network as downstream target of ACHT4 (35). During dark-light transitions, the fast reoxidation of ACHT4 depends on 2-CysPrx. 2-CysPrx likely functions as oxidant to switch off starch synthesis in the dark similar to the proposed hypothesis that 2-CysPrx acts as a local redox sink transferring electrons to ROS to shut down photosynthesis. There is a need to kinetically resolve the redox state of components of the thiol-disulfide redox regulatory network in WT and mutants to better define the role of 2-CysPrx in rapid redox regulation (45).

Recently, an ex vivo approach based on coimmunoprecipitation (CoIP) and mass spectrometry allowed for identifying a set of 2-CysPrx interactors (20). The strength of this study rested on the comparison of CoIP proteomes of WT and 2CysPrxA/B-deficient $A$. thaliana. Polypeptides recovered with anti-2CysPrx antibody from 2 cysprx $A / B$-CoIPs were considered as false positives and subtracted from the polypeptides detected in WT CoIPs. This approach identified 67 interacting polypeptides from the chloroplast. The 91 interactions with polypeptides from extraplastidic compartments may originate from lost compartmentation during extraction. Fructose-1,6bisphosphatase, ACHT2, and CDSP32 were among the known interactors. Surprisingly, Trx, NTRC, and Cyp20-3 were not found possibly because of the weak nature of interactions.

Interestingly, binding to other cyclophilins such as Cyp37/ 38 and CLP protease supports a role of 2-CysPrx protein in protein folding or regulation. The interactions with, for example, protochlorophyllide reductase B, 5-adenylylsulfate reductase (APR), GSH reductase, and three ferredoxin (Fd)dependent proteins, namely $\mathrm{Fd}$-sulfite reductase, Fd-nitrite reductase, and Fd-glutamate synthase reinforce the suggested function of 2-CysPrx in the redox-regulatory network of the chloroplast (20). Future work will have to address the significance of the interactions for interactor function and chloroplast metabolism.

A prerequisite for protein interactions are partner-specific surface-exposed residues, which form the interface either permanently or become surface exposed after conformational changes induced by interaction or variation of the milieu. A 2-CysPrx site with binding characteristics is the flexible Cterminus that is involved in binding the Ser protease Omi/ HtrA 2 and embraces Srx 1 during the retroreduction of the hyperoxidized sulfinylated 2-CysPrx $(50,56)$. As shown for the interaction of At2-CysPrx with NTRC, the deletion of the C-terminus decreases the affinity for NTRC and alters the peroxidase and chaperone activities (59). The best studied and closest sequence homologues of At2-CysPrx likely are human Prdx1 and Prdx2. Analysis of their interactomes allowed the identification of the sequences CXXC, PXXP, and LXXL as motifs found in either Prdx1- or Prdx2-binding partners (15).

Coexpression frequently allows pinpointing to functional relationships. Transcriptional coexpression with Prxs has been reported by Muthuramalingam et al. (77) and Dietz (31) and identified Cyp20-3 as best hit for coexpression with values of $0.97-0.99$. Here we extended this analysis to include the transcriptome of Oryza sativa $c v$. japonica (Rice Oligonucleotide Array Database [ www.ricearray.org]) in comparison with $A$. thaliana (taken from ATTED-II [http:// atted.jp]). The obtained sets of highly coexpressed genes showed a significant match between Arabidopsis and rice for 2-CysPrx or PrxIIE. Sets of $61 \%$ for 2-CysPrx and $45 \%$ for PrxIIE of the identified Arabidopsis genes were also highly coregulated in rice (correlation coefficient $>0.5$ ) (Fig. 5). Strong connections to photosynthesis, antioxidant partners, and ribosomal activity were seen in both species. The comparison is still constrained by the incomplete annotation of the rice genome, possibly causing an underestimation of the coregulated genes, but also limiting the differentiation of the Arabidopsis and rice response.

\section{Post-Translational Effects}

The cysteine oxidation is the prime PTM on Prxs. The robust Trx-fold, conserved motifs, and flexible structures mediate conformational changes along with redox-dependent thiol modification. Apart from their thiol peroxidase function, Prxs can be considered as a prototypic thiol switch protein that is based on present-day knowledge evolved early during evolution. The dithiol-disulfide transition modifies conformation and function. Nowadays, the thiol switch mechanism is recognized for its broad significance in plants and other organisms with oxygenic metabolism (110). Such thiol switch proteins play fundamental roles in regulation and signaling of eukaryotes and prokaryotes, including mitochondria $(4,5,40,44,79)$. ROS are generated at low rates by metabolism and, for example, by NADPH oxidases under normal conditions. They are detoxified by antioxidant enzymes, including thiol peroxidases (32). Thiol peroxidases are regenerated by redox transmitters, which then may oxidize 

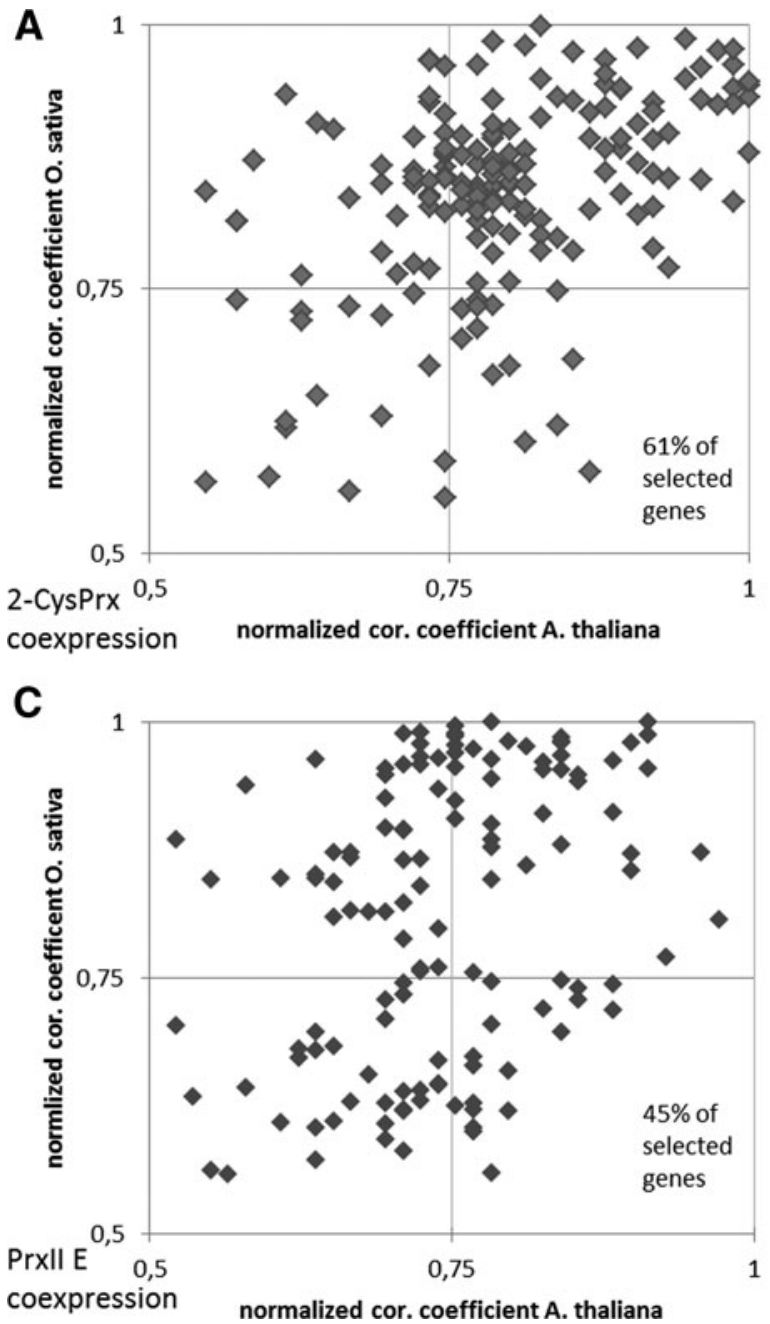

B Highlighted genes coexpressed with 2-CysPrx

\begin{tabular}{|c|c|c|}
\hline gene function & A.thaliana rank & O. sativa rank \\
\hline Photosystem II oxygen-evolving complex & 81 & 112 \\
Photosystem II reaction center PSB29 protein & 26 & 40 \\
Uroporphyrinogen decarboxylase & 57 & 20 \\
\hline Heavy metal transport/detoxification family protein & 34 & 56 \\
RABGTPase homolog E1B & 29 & 5 \\
\hline Ribosomal protein L21 & 2 & 18 \\
Ribosomal protein L13 family protein S20 & 16 & 16 \\
Ribosomal protein L10 family protein & 13 & 7 \\
Phosphoglycerate kinase 1 & 12 & 6 \\
Chloroplast 30S ribosomal protein S20 & 3 & 9 \\
\hline Coproporphyrinogen III oxidase & 65 & 172 \\
PhotosystemIP subunit & 35 & 105 \\
Peroxiredoxin Q & 17 & 90 \\
Copper/zincsuperoxidedismutase & 182 & 73 \\
O-acetylserin-(thiol) lyase B & 138 & 10 \\
\hline RNA-binding (RRM/RBD/RNP) family protein & 22 & 88 \\
CLP protease P4 & 27 & 84 \\
\hline Cyclophilin 20-2 & 19 & 2 \\
Cyclophilin 20-3 & 1 & 145 \\
\hline
\end{tabular}

D

Highlighted genes coexpressed with Prxll E

\begin{tabular}{|c|c|c|}
\hline gene function & A.thalianarank & 0. sativarank \\
\hline FAD/NAD(P)-binding oxidoreductase & 32 & 10 \\
Uroporphyrinogen decarboxylase & 63 & 38 \\
Photosystem II reaction center PSB29 protein & 38 & 44 \\
Photosystem II reaction center PSB28 protein & 36 & 23 \\
Chaperonin 60 beta & 76 & 81 \\
Peroxiredoxin Q & 10 & 24 \\
Thioredoxin 2 & 89 & 120 \\
Ribosomal protein L21 & 4 & 2 \\
Plastid ribosomal protein I11 & 5 & 7 \\
Chloroplast 30S ribosomal protein S20, putative & 7 & 12 \\
Ribosomal protein L17family protein & 20 & 13 \\
Translationelongation factor EF1B & 19 & 11 \\
\hline Dicarboxylate transporter 1 & 17 & 89 \\
Glutamate-1-semialdehyde 2,1-aminomutase 2 & 9 & 61 \\
Chloroplast outer envelope protein 37 & 130 & 49 \\
Long chain acyl-CAA synthetase9 9 & 132 & 50 \\
YGGT family protein & 1 & 74 \\
Thioesterase superfamily protein & 44 & 133 \\
CLP protease P4 & 12 & 86 \\
2-Cysteineperoxiredoxin & 2 & 55 \\
\hline Cyclophilin 20-2 & 8 & 56 \\
Cyclophilin 20-3 & 16 & 87 \\
\hline
\end{tabular}

FIG. 5. Transcripts coexpressed with 2-CysPrx and PrxIIE in Arabidopsis thaliana and Oryza sativa. The correlation coefficients for $O$. sativa and $A$. thaliana were obtained from the ATTED-II and the Rice Oligonucleotide Array Database $(6,19)$. Correlation coefficients were normalized and ranked with 1 for the transcript with maximum level of coregulation and higher numbers according to the position in the list. The query list comprised the Arabidopsis genes with a correlation $>0.5$. The homologous genes were searched in rice, the degree of coexpression with 2-CysPrx and PrxIIE was determined and plotted against the corresponding coefficient of Arabidopsis. (A) The transcripts in the area with high correlation of $>0.5$ in both rice and Arabidopsis represent $61 \%$ of total analyzed genes, indicating a high level of similarity in coregulation in both species. (C) For PrxIIE, 45\% of the selected genes ranged in the area of high coexpression in both species. A selection of interesting genes from this group is given for 2-CysPrx (B) and PrxIIE (D)

other thiol switch proteins. Accumulating ROS under stress in addition may target sensitive thiols of other proteins (76).

Reversibility of disulfide formation, sulfenylation, and possibly sulfinylation contrasts sulfonylation and oxidative modifications of other amino acid residues, which often mark proteins for degradation $(10,28)$. Thus it was a very important finding that the hyperoxidized sulfinic acid forms of 2CysPrx and PrxIIF can be regenerated by Srx $(16,53,118)$. The specificity or promiscuity of Srx in reduction of sulfinylated proteins still needs to be scrutinized because hyperoxidation of 1-CysPrx may be irreversible (118). These authors state that hyperoxidized 1-CysPrx but also glyceraldehyde-3phosphate dehydrogenase might be degraded because neither of them is a target of Srx.

Redox-dependent inhibition of Prx peroxidase activity triggers alternative functions and signaling pathways. Hyperox- idized 2-CysPrx prevents heat-induced denaturation of citrate synthase in vitro $(54,59)$. Overexpression of 2-CysPrx suppresses aggregation of cyclophilin 20-3 coexpressed in protoplasts in vivo (77). Combining redox-dependent peroxidase and chaperone function in a highly abundant protein provides the opportunity to operate a molecular redox switch in response to oxidative stress. The functional switch from peroxidase to chaperone is part of the floodgate model. Hyperoxidation of Prxs might shut down the detoxification in the thiol peroxidase branch and temporally and spatially enhance the ROSdependent signaling (121). Analysis of 2-cysprx $A / B$ knockout mutants revealed enhanced photo-oxidative damage and higher $\mathrm{H}_{2} \mathrm{O}_{2}$ levels in high light. Considering its high abundance in chloroplasts and its association with the thylakoids, the 2CysPrx might act as a local redox-controlled switch to allow for the release of ROS signals from the photosynthetic electron 
transport chain. In turn, ROS release may change chloroplast processes but also initiate retrograde control of extrachloroplast processes. However, regulation of ROS-responsive marker genes such as ZAT12, BAP1, HSFA2, or OXI1 was unaltered in 2 cysprx $A / B$ compared with WT and only responded after introducing the additional knockout in the thylakoid ascorbate peroxidase $(t A p x)$ gene. This might indicate a synergistic floodgate control by thiol and ascorbate peroxidases and mutual compensation of the lost other enzyme (9).

PTMs of Prxs by glutathionylation, nitrosylation, acetylation, or phosphorylation partly mimic cysteine hyperoxidation and thus oligomerization, and realize oxidation-independent regulation and stress responses. The tripeptide GSH is present at millimolar concentrations in plasmatic compartments and functions as redox-thiol buffer, electron donor, substrate for phytochelatin synthesis, and in $S$-glutathionylation (80). $S$-glutathionylation is necessary for redox regeneration of 1-CysPrx and type II Prxs, which then depends on GST or Grxcoupled cysteine reduction.

Under conditions of GSSG accumulation, the mass of PsTrx-h2 increases to $14,361.1$ Da corresponding to addition of 305.5 Da of GSH to 14,056.9 Da (42). Another example of reversible glutathionylation occurs with chloroplast glyceraldehyde-3-phosphate dehydrogenase and NADP-dependent malate dehydrogenase $(13,69)$. The GSH/GSSG pool participates in redox homeostasis and controls various signaling pathways. Reversible $S$-glutathionylation introduces a negative charge and a bulky tripeptide and protects thiols from hyperoxidation.

Peroxynitrite $\left(\mathrm{ONOO}^{-}\right)$serves as substrate for 2-CysPrx and PrxIIE (8, 107). Biotic and abiotic stress conditions provoke nitrosoglutathione (GSNO) accumulation, which is a storage pool for NO. NO is a radical and participates in cell signal transduction (38). A proteome analysis identified $>1000 S$-nitrosylated targets in A. thaliana and likewise in Kalanchoe pinnata, Brassica juncea, and Citrus aurantium. These proteins are involved in major cellular activities such as primary and secondary metabolism, photosynthesis, genetic information processing, cellular architecture, and response to biotic and abiotic stresses (8). As part of detoxification networks, PrxIIE and PrxIIB (cytosol), PrxIIF (mitochondria), and GR and Gpx are targets of $S$-nitrosylation. These and other nitrosylated polypeptides were inventoried by MS/MS analysis after addition of GSNO to Arabidopsis cell cultures or exposing leaves to $1 \mathrm{ppm}$ NO via the gaseous phase (63). Proof of physiological significance is scarce. $S$-nitrosylation of PrxIIE is suggested to ease ONOO-dependent tyrosine nitration as signaling event in pathogen defense (104). Plant Prxs are Lys acetylated as reported for Os1-CysPrx (47) and for Os2-CysPrx (124). Acetylation sites are KSGGLGDLK(ac)TK, GLFIIDK(ac)HSTINNLAIGR, and SISK(ac)GVLIPDQGIALR in Os2-CysPrx. Lys acetylation in mammalian astrocytes was connected to the oxidation state of Cys residues because many acetylation sites are detected in the vicinity of oxidized cysteines in cell lines depleted of most of their GSH (88). Human Prdx2 is subjected to $\mathrm{N}$-terminal acetylation, which protects the catalytic cysteine from hyperoxidation to irreversible sulfonic acid, retaining peroxidase activity by Srx regeneration (109). The physiological role of acetylation awaits investigation in plants.

More is known about the physiological role of plant protein phosphorylation $(98,102,115,126)$. A recent analysis of the phosphoproteome of grapevine (67) confirmed phosphorylation of $\mathrm{Vv}$-Apx (Vitis vinifera) in addition to the cytoplasmic VvPrxIIB in response to phytoplasma infection. A similar pattern was found in A. thaliana after wounding and in Medicago truncatula after rhizobial infection $(52,105)$. To

Table 2. Phosphorylation Sites of Peroxiredoxins and Selected Redox Proteins

\begin{tabular}{|c|c|c|c|}
\hline Protein & Protein ID & $p$-site & Literature \\
\hline AtPrxIIE & Q949 U7 & $\begin{array}{l}{ }^{80} \mathrm{PDsTL}^{84} \\
{ }^{129} \mathrm{FVsKA}^{133}{ }^{13}\end{array}$ & $\begin{array}{c}7 \\
103\end{array}$ \\
\hline AtPrxIIF & Q9 M7T0 & $\begin{array}{l}{ }^{35} \mathrm{EGtDI}^{39} \\
{ }^{51} \mathrm{ARsWD}^{55} \\
{ }^{138} \mathrm{EFyGD}^{142} \\
{ }^{147} \mathrm{HKsLG}^{151}\end{array}$ & $\begin{array}{c}7 \\
103 \\
123 \\
7\end{array}$ \\
\hline AtPrxQ & Q9LU86 & ${ }^{138} \mathrm{DDsAS}^{142}$ & 103 \\
\hline $\begin{array}{l}\text { At2- } \\
\text { CysPrxA }\end{array}$ & Q96291 & $\begin{array}{l}{ }^{96} \mathrm{KLsDY}^{100} \\
{ }^{159} \mathrm{RKsGG}^{163} \\
{ }^{208} \mathrm{HStIN}^{212} \\
{ }^{257} \mathrm{KLsKEY}^{262}\end{array}$ & $\begin{array}{c}103 \\
114 \\
103,114 \\
103\end{array}$ \\
\hline $\begin{array}{l}\text { At2- } \\
\text { CysPrxB }\end{array}$ & Q9C5R8 & $\begin{array}{l}{ }^{214} \mathrm{QHsTI}^{218} \\
{ }^{215} \mathrm{HStIN}^{219} \\
{ }^{264} \mathrm{KLsKEY}^{269}\end{array}$ & $\begin{array}{c}114 \\
103,114 \\
103\end{array}$ \\
\hline $\begin{array}{l}\text { Mt2- } \\
\text { CysPrxB }\end{array}$ & G7ZUV5 & $\begin{array}{l}{ }^{158} \mathrm{RKsGG}^{162} \\
{ }^{179} \mathrm{SKsYG}^{183}\end{array}$ & $\begin{array}{l}105 \\
105\end{array}$ \\
\hline AtCDSP32 & Q9SGS4 & $\begin{array}{l}{ }^{117} \text { ELsRT }^{121} \\
{ }^{233} \text { SRsMS }^{237} \\
{ }^{237} \text { SMsET }^{241}\end{array}$ & $\begin{array}{c}70 \\
114 \\
99,103,114\end{array}$ \\
\hline AtACHT1 & Q8LEK4 & ${ }^{202}$ VGsIS $^{206}$ & 103 \\
\hline AtTrx-m2 & Q9SEU8 & ${ }^{178}$ TSsLD $^{182}$ & 37 \\
\hline AtTrx-m4 & Q9SEU6 & $\begin{array}{l}{ }^{156} \text { IRsVP }^{160} \\
{ }^{171} \mathrm{KDsII}^{175}\end{array}$ & $\begin{array}{l}103 \\
103\end{array}$ \\
\hline AttAPX & Q42593 & $\begin{array}{l}{ }^{79} \mathrm{AAsDA}^{83} \\
{ }^{213} \mathrm{PPsPA}^{217} \\
{ }^{371} \mathrm{SDsMK}^{375}\end{array}$ & $\begin{array}{c}70 \\
103 \\
103\end{array}$ \\
\hline MttAPX & MEDTR3G088160 & $\begin{array}{l}{ }^{228} \mathrm{PpsPA}^{232} \\
{ }^{255} \mathrm{AHtLG}^{259} \\
{ }^{367} \mathrm{DGsPN}^{371}\end{array}$ & $\begin{array}{l}105 \\
105 \\
105\end{array}$ \\
\hline AtsAPX & Q42592 & $\begin{array}{l}{ }^{234} \text { PPsPA }^{238} \\
{ }^{237} \text { PAtHL }^{241}\end{array}$ & $\begin{array}{c}78,116,117 \\
116\end{array}$ \\
\hline MtAPX1 & G7JRI5 & $\begin{array}{l}{ }_{5}^{2} \mathrm{GKsYP}^{6} \\
{ }^{5} \mathrm{YPtVS}^{9} \\
{ }_{47} \mathrm{FDsKT}^{51} \\
{ }^{211} \mathrm{LLsDP}^{215} \\
{ }^{241} \mathrm{KLsEL}^{245}\end{array}$ & $\begin{array}{l}105 \\
105 \\
105 \\
105 \\
105\end{array}$ \\
\hline MtAPX4 & MEDTR5G064610 & ${ }^{157}$ GLsAA $^{161}$ & 105 \\
\hline AtGPX1 & P52032 & ${ }^{176}$ GPsTA $^{180}$ & 123 \\
\hline AtGPX2 & O04922 & ${ }^{165}$ GQAss $^{169}$ GQASs $^{169}$ & $\begin{array}{l}128 \\
103\end{array}$ \\
\hline AtFD1 & O04090 & $\begin{array}{l}{ }^{25} \mathrm{LRsLP}^{29} \\
{ }^{40} \mathrm{LKsTAR}^{45} \\
{ }^{41} \mathrm{KStAR}^{45}\end{array}$ & $\begin{array}{l}70 \\
70 \\
70\end{array}$ \\
\hline
\end{tabular}

Phosphorylation sites (p-site) with phosphorylated serine, threonine, and tyrosine (small letters $\mathrm{s}$, $\mathrm{t}$, y in bold) of peroxiredoxins, thioredoxins, ascorbate peroxidases, glutathione peroxidases, CDSP32, and ACHT1 revealed by employing databases mentioned on the next page $(48,127)$. The sequence containing the modified aminoacid (aa) was arbitrarily set 5-6 aa long. Aminoacid positions refer to primary sequence of the proteins as counted from $\mathrm{N}$ to $\mathrm{C}$ terminus with transit peptide included. Following proteins were queried: superoxide dismutase, PrxIIE, PrxIIF, PrxQ, 2-CysPrx A, 2CysPrx B, Apx, ascorbate peroxidase, tApx, thylakoid ascorbate peroxidase, sApx, stromal ascorbate peroxidase; chloroplast Gpx, chloroplast; Trx members (Trx-f1, -f2, m1-4, -x, -y1, -y2,-z), Trx-like proteins (NTRC, CDSP32, ACHT 1-4 [atypical cysteine histidinerich Trx], and APR 1-3 [protein disulfide isomerase-like proteins]), Fd, ferredoxin 1; FNR, ferredoxin-dependent NADPH reductase 1.

$\mathrm{P}$-sites were found in the cited references. 
address the consequences of kinase inhibition, Xu et al. (125) observed reduction in the drought-induced $\mathrm{H}_{2} \mathrm{O}_{2}$ production and a decrease in the drought-enhanced activities of antioxidant enzymes such as SOD, Apx, and Gpx in maize. Queries to the online databases IPTM net (http://research.bioinformatics .udel.edu/iptmnet), P3DB (www.p3db.org), and PhosPhat 4.0 (http://phosphat.uni-hohenheim.de) retrieve serine/threonine protein kinases involved in modifying redox proteins. Of the redox proteins mentioned in this review, PrxIIE, PrxIIF, PrxQ, 2-CysPrxA, 2-CysPrxB, CDSP32, ACHT 1, Trx-m4, Apx, and Gpx are found to be phosphorylated among others (Table 2). The table provides detailed information on the identified phosphorylated peptides of the redox proteins.

Interestingly, At2-CysPrx and the mammalian 2-CysPrx type Prdx 1 have the C-terminal SKEYF site in common, which is phosphorylated at the Ser in A. thaliana and at the Tyr in mouse, rat, and human (119). In vitro phosphorylation of Tyr in the respective site of human Prdx1 decreases the peroxidase activity by $80 \%$ (119). According to their proposed model, a local rise in peroxide concentration leads to MAPK activation, and phosphorylation of peroxiredoxin, in turn, allows for further signaling. Whether this model is applicable to plant 2CysPrxs and other redox proteins is not known. Altogether, these results indicate a relationship between redox regulation, PTMs, and signaling. The PTM of redox proteins and their implications in planta still needs to be explored.

\section{Membrane Interaction}

The C-terminus of 2-CysPrx bears the motif SKEYF, which is conserved among plant 2-CysPrx but absent in its prokaryotic homologue, the alkyl hydroperoxide reductase $\mathrm{C}$ (AhpC). In addition to the feature of SKEYF phosphorylation mentioned in the previous section, the SKEYF motif is also present in the Cterminal sequence ${ }^{186}$ NVDDSKEYFSKHN ${ }^{198}$ of the human 2Cys Prx, Prdx2, where it mediates its membrane attachment (22). Therefore, as shown for barley 2-CysPrx, the C-terminus that is essential for binding to NTRC (59) likely plays a role in 2-CysPrx membrane association, in regulation of its peroxidase activity (see previous section), or both. The SKEYF-containing sequence ${ }^{184}$ EKSMKPDPKLSKEYFSAI $^{201}$ is computationally predicted to interact with membranes (Table 3). A different approach to investigate the possibility of peripheral membrane association of 2-CysPrx considers electrostatic interactions between the decamer and the membrane (68). Here it is striking that the outer pentagon sites of 2-CysPrx bear Lys-rich Ctermini and the inner doughnut sites are enriched in positively charged residues. Yet, it is unknown whether these features play a role in realizing the putative amphitropic character of 2CysPrx, wherein barley 2-CysPrx is found both in the soluble plastid proteome and associated with the thylakoid (58). Amphitropic proteins reversibly interact with the membrane by lipid-binding regions containing positively charged and hydrophobic amino acids residing in coil-helical regions $(18,55)$. Hydrophobic faces are also often involved in membrane interactions (41) and the analysis shown in Table 3 revealed a hydrophobic face sequence ${ }^{162}$ LQALQYIQENPDEVCPAG ${ }^{179}$. The hydrophobic face has negatively charged residues opposed to their hydrophobic domains, which could play a role in At2CysPrx membrane association as proposed for the human Prdx2 membrane interaction model (92). Protons or $\mathrm{Ca}^{2+}$ ions may neutralize the negatively charged hydrophobic faces to imbed 2-CysPrx in the membrane.

The Aramemnon online tool (http://aramemnon.uni-koeln. de/request.ep) was then used to explore the lipid-binding characteristics of 2-CysPrx (108). A lipid anchor modification was not predicted, but a weak transmembrane helical region was assigned to the ${ }^{37}$ GKKYVILFFYPLDFTFVC $\mathrm{PTE}^{57}$ sequence. Support for this sequence motif as putative site of membrane binding comes from the observation that the contained ILFF motif is present in membrane-associated proteins such as membrane-associated protein (At1 g80910), Gibberellin receptor (At3 g05120), and GDSL lipase (At1 g54790). The ILFF motif is also present in the phosphatidic acid-binding site of Raf $1, \mathrm{~A}-\mathrm{Raf}$, and B-Raf $(2,43)$. In the human 1-CysPrx Prdx6 that has both lipase and peroxidase activities, the ILF motif is present in its $\alpha / \beta$ hydrolase fold (65). Of the three putative membrane interaction sites, the ${ }^{184}$ EKSMKPDPKLSKEYFSAI ${ }^{201}$ and ${ }^{37}$ GKKYVILFFYPL DFTFVCPTE $^{57}$ sites are rich in Lys content and are present on both halves of the decameric donut (Fig. 6). The latter site is suggested to contain a binding groove for lipid peroxide substrates. The site starting with the ${ }^{35} \mathrm{YIGKK}^{39}$ loop and ending with the ${ }^{49} \mathrm{DFTFV}^{53}$ loop contains the $\mathrm{Cys}_{\mathrm{P}}$, is highly hydrophobic, and is proposed to have a length of $\sim 27 \AA$ and a width spanning from 4 to $12 \AA$ resembling the lengths of known lipid-binding proteins such as $\beta$-lactoglobulin (PDB ID: 1EXS) or hPrdx6 (PDB ID: 1PRX) (Fig. 6B).

The $\mathrm{Ca}^{2+}$-associated protein calpromotin nowadays called Prdx2 in mammals interacts with integral membrane proteins such as the gardos channel (Uniprot entry O15554), stomatin (Uniprot entry P27105), and band 3 (Uniprot entry P02730) $(68,73,74)$. In contrast to Cha et al. (22), Matte et al. (68) proposed that the $\mathrm{N}$-terminus of cytosolic band $3(\mathrm{Cdb} 3)$ is crucial for Prx 2 membrane association, whereas Plishker et al. (92) assume that the redox milieu and $\mathrm{Ca}^{2+}$ are essential for the switch from the soluble to the membrane-bound state. Alternatively, binding proteins may mediate thylakoid attachment of 2-CysPrx. Muthuramalingam et al. (77) detected 2-CysPrx in photosystem II-enriched fractions and were able

Table 3. At2-CysPrx Sequences with $\alpha$-Helical Characteristics, Analyzed with HELIQUEST

\begin{tabular}{llrrr}
\hline Name & Sequence & $z$ & $<\mathrm{H}>$ & $<\mu \mathrm{H}>$ \\
\hline At2-CysPrx 162-179 & LQALQYIQENPDEVCPAG & -3 & 0.426 & 0.337 \\
At2-CysPrx 184-201 & EKSMKPDPKLSKEYFSAI & 1 & 0.172 & 0.418 \\
\hline
\end{tabular}

The analysis with the HELIQUEST program used the default settings. Regions of At2-CysPrx were selected with minimum $50 \% \alpha$ helical character, but no $\beta$-strand content and no dimer-dimer interaction site (120). Secondary structure features of At2-CysPrx are based on the Phyre 2 (www.sbg.bio.ic.ac.uk/phyre2) alignment with human peroxiredoxin as template (1qmv, 62\% identity, 100\% confidence). Sequences with putative lipid-binding helices and hydrophobic faces (a) are presented. According to the discrimination factor equation $\mathrm{D}=0.944$ $(<\mu \mathrm{H}>)+0.33(\mathrm{z})$, possible lipid-binding helices exist, when the calculated discrimination factor D lies between 0.68 and $1.33(41)$. 


\section{A}

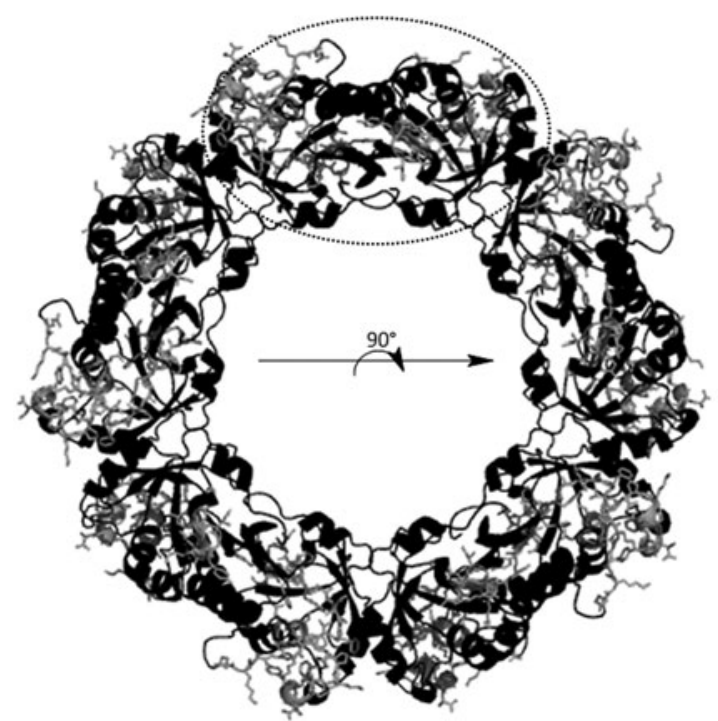

B

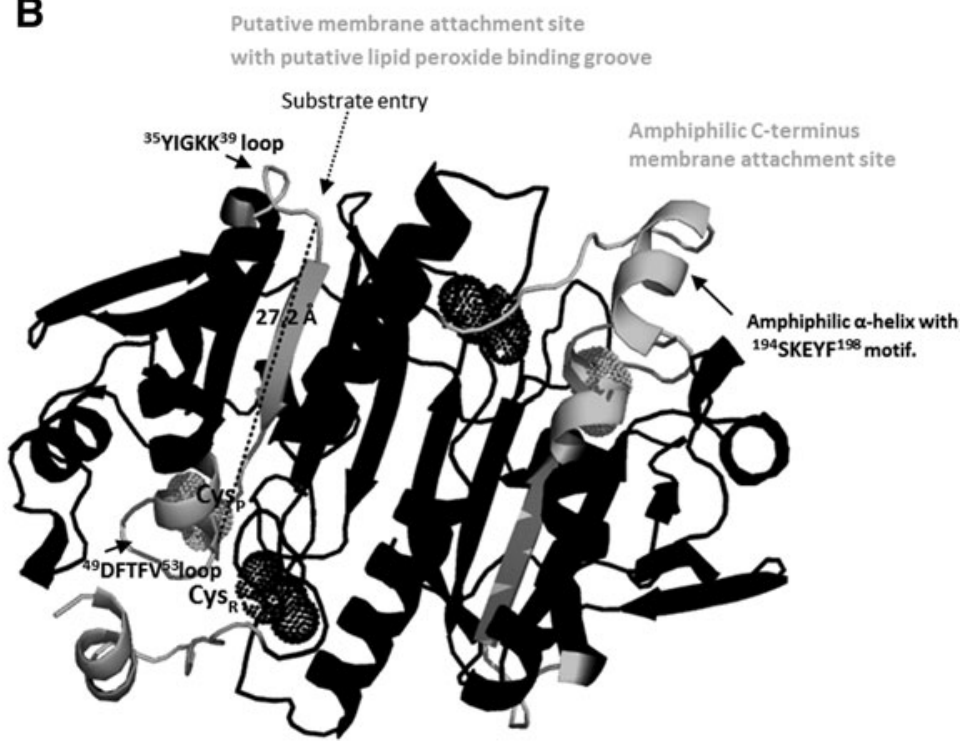

FIG. 6. Putative membrane attachment sites of At-2-CysPrx. (A) 2-CysPrx pentadimer with membrane attachment sites highlighted in gray and residing residues shown as stick models. Each subunit contains two membrane attachment sites. The 3D model was constructed with Phyre 2 (www.sbg.bio.ic.ac.uk/ phyre) and PyMOL (29). hPrx2 (PDB ID 1qmv, $62 \%$ identity) was used as template (57). (B) $90^{\circ}$ turn of 2-CysPrx pentadimer as indicated with viewpoint on the indicated dimer (dotted circle) after rotation. Each monomer is suggested to mediate membrane attachment of one homodimer via two sites (highlighted in gray), the C-terminus and the loop surrounding the substrate entry site. Stick models of residues visible in $(\mathbf{A})$ are not shown in (B) in order to ease the perspective on the lipid peroxide-binding groove (length as dotted line in Angstrom $[\AA]$ ). The peroxide-binding groove starts (amino acids counted from the N-terminus) at the positively charged Lys-rich loop near the entrance (aa 35-39), proceeds to the ${ }^{42} \mathrm{ILF}^{44}$-motif of the $\beta$-strand, and ends at the ${ }^{49} \mathrm{DFTFV}^{53}$ loop close to the $\mathrm{Cys}_{\mathrm{P}}$ (dotted spheres) of the cavity. The resolving Cys of the adjacent monomer is shown as dotted black sphere.

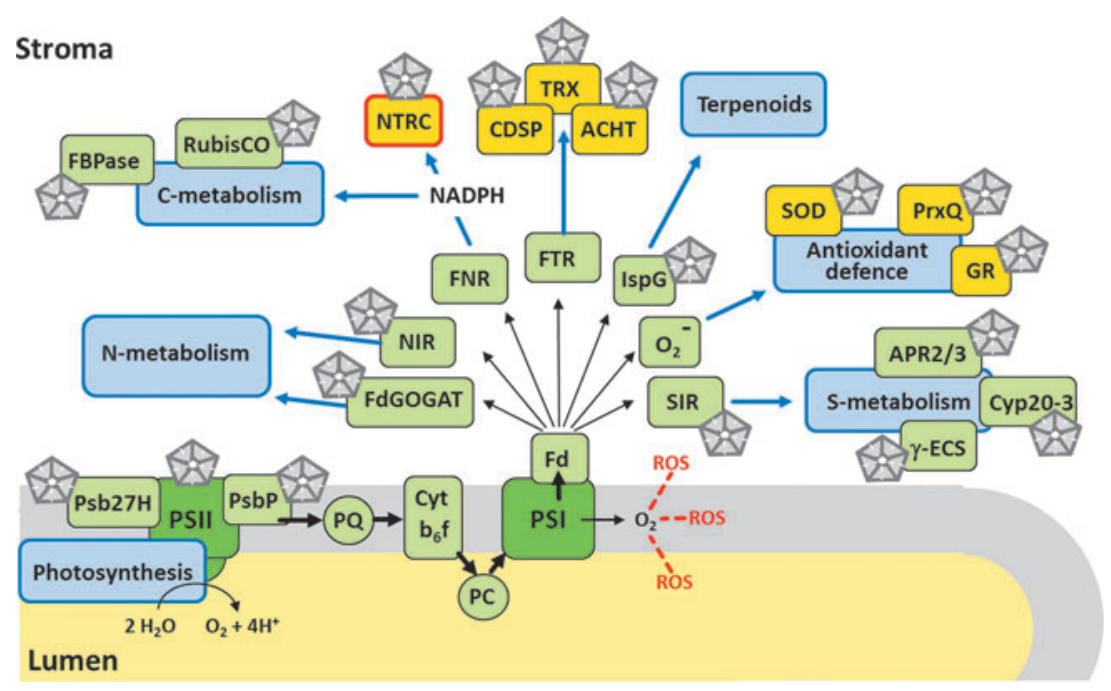

FIG. 7. Assignment of the 2-CysPrx interacting partners to selected metabolic pathways of the chloroplast. The figure is based on diverse findings, in particular, the interactome article by Cerveau et al. (20) and focuses on the photosynthetic electron transport and the associated ferredoxin $(\mathrm{Fd})$, which distributes electron via diverse Fd-dependent proteins to various reductive pathways. The pentagon icon indicates interaction of 2-CysPrx with the proteins. See text for further discussion. ACHT, atypical chloroplast thioredoxin; APR, adenylylsulfate reductase; Cyp20-3, cyclophilin 20-3; $\gamma$-ECS, gamma glutamyl cysteine synthase; FBPase, fructose-1,6-bisphosphatase; Fd-GOGAT, ferredoxin-dependent glutamate synthase; FNR, ferredoxin-dependent NADPH reductase; FTR, ferredoxin-dependent thioredoxin reductase; GR, glutathione reductase; IspG, ferredoxin-dependent 4-hydroxy-3-methylbut-2-en-1-yl diphosphate synthase; NIR, ferredoxindependent nitrite reductase; PC, plastocyanine; PQ, plastoquinone; Psb, photosystem II; PSI/II, photosystem I/II; RubisCO, ribulose-1,5-bisphosphatase; SIR, ferredoxin-dependent sulfite reductase; SOD, superoxide dismutase. 
to coimmunoprecipitate D1 protein and 2-CysPrx. Prdx2 is highly similar to At2-CysPrx. Therefore, one may ask whether the binding epitopes of the mentioned proteins can be found in At2-CysPrx. A protein Blast (https://blast.ncbi.nlm. nih.gov/Blast.cgi?PAGE=Proteins) search of the $A$. thaliana uniprot databank with the gardos channel retrieved two pore domain potassium channels, the vacuolar AtTPK1 (At5 g55630) and the plasmamembrane AtTPK4 (At1 g02510). They share the GYGD consensus motif with the gardos channel and are $\mathrm{Ca}^{2+}$-activated $\mathrm{K}^{+}$channels but reside in a different compartment than the plant 2-CysPrx. The A. thaliana genome contains genes encoding stomatin-like proteins (33). Future studies focusing on the interaction of 2-CysPrx with integral membrane proteins will be of great interest.

\section{Final Remarks}

Research on plant Prxs began with the identification of 2CysPrx in the chloroplast and 1-CysPrx in the nucleus 20 years ago $(11,113)$. Since then, the biochemical features, cell biological mechanisms, and physiological implications of different plant Prxs have been elucidated from various perspectives, but the most significant advancement has been achieved for the chloroplast 2-CysPrx either by targeted investigation or by coincidental findings, for example, by identifying NTRC as most efficient but not exclusive electron donor of 2-CysPrx. Since the last comprehensive review (31), significant progress has been achieved in identifying the interactome as selectively displayed in Figure 7 for 2-CysPrx (20). Key components of the major metabolic pathways that consume reductive power have been proven or are suggested to interact with 2-CysPrx. This concerns photosynthetic light reactions, carbon metabolism, nitrogen and sulfur metabolism, antioxidant defense, and secondary compound synthesis. In this context, it is intriguing that ferredoxin-dependent proteins constitute a major fraction of interacting partners. Important interactions with proteins or membranes need to be confirmed and explored with respect to binding selectivity for redox-dependent conformational states. The successful proteomics analysis indicates that there is a need to conduct metabolomics and transcriptomics analyses to reach the global assessment of the physiological significance of the different Prxs. This includes the interference with $\mathrm{Ca}^{2+}$ and hormone signaling. Most importantly we need complementation of the 2-CysPrx knockout line with variants that mimic specific states of 2-CysPrx, namely devoid of peroxidase activity, lacking the oligomerization ability, resembling the hyperoxidized decamer, or with truncated C-terminus. It will be interesting to find out which features of the knockout phenotype can be complemented by each of the variants and this approach should allow dissecting the roles as thiol peroxidase, oxidant, interaction partner, and chaperone.

\section{Acknowledgment}

The work in this article was supported by the Deutsche Forschungsgemeinschaft DI346/14 and SPP1710.

\section{References}

1. Altschul SF, Gish W, Miller W, Myers EW, and Lipman DJ. Basic local alignment search tool. J Mol Biol 215: 403-410, 1990.
2. Andresen BT, Rizzo MA, Shome K, and Romero G. The role of phosphatidic acid in the regulation of the Ras/ MEK/Erk signaling cascade. FEBS Lett 531: 65-68, 2002.

3. Anschau V, Ferrer-Sueta G, Portillo-Ledesma S, and Netto LES. Kinetic Characterization of Sulfenic Acid Reduction in 1-Cys Peroxiredoxins by Ascorbate. In Annual Meeting of the Society for Free Radical Biology and Medicine (SFRBM). Soc Free Radic Biol Med 76: S138, 2014.

4. Antelmann H. Enzyme regulation: a thiol switch opens the gate. Nat Chem Biol 11: 4-5, 2015.

5. Antelmann $\mathrm{H}$ and Helmann JD. Thiol-based redox switches and gene regulation. Antioxid Redox Signal 14: 1049-1063, 2011

6. Aoki Y, Okamura Y, Tadaka S, Kinoshita K, and Obayashi T. ATTED-II in 2016: a plant coexpression database towards lineage-specific coexpression. Plant Cell Physiol 57: e5, 2016.

7. Aryal UK, Krochko JE, and Ross AR. Identification of phosphoproteins in Arabidopsis thaliana leaves using polyethylene glycol fractionation, immobilized metal-ion affinity chromatography, two-dimensional gel electrophoresis and mass spectrometry. J Proteome Res 11: 425437, 2011.

8. Astier J, Kulik A, Koen E, Besson-Bard A, Bourque S, Jeandroz S, Lamotte O, and Wendehenne D. Protein Snitrosylation: what's going on in plants?. Free Radic Biol Med 53: 1101-1110, 2012.

9. Awad J, Stotz HU, Fekete A, Krischke M, Engert C, Havaux M, Berger S, and Mueller MJ. 2-cysteine peroxiredoxins and thylakoid ascorbate peroxidase create a water-water cycle that is essential to protect the photosynthetic apparatus under high light stress conditions. Plant Physiol 167: 1592-1603, 2015.

10. Bader $\mathrm{N}$ and Grune T. Protein oxidation and proteolysis. Biol Chem 387: 1351-1355, 2006.

11. Baier M and Dietz KJ. Primary structure and expression of plant homologues of animal and fungal thioredoxindependent peroxide reductases and bacterial alkyl hydroperoxide reductases. Plant Mol Biol 31: 553-564, 1996.

12. Barranco-Medina S, Krell T, Bernier-Villamor L, Sevilla F, Lázaro JJ, and Dietz KJ. Hexameric oligomerization of mitochondrial peroxiredoxin PrxIIF and formation of an ultrahigh affinity complex with its electron donor thioredoxin Trx-o. J Exp Bot 59: 3259-3269, 2008.

13. Bedhomme M, Adamo M, Marchand $\mathrm{CH}$, Couturier J, Rouhier N, Lemaire SD, Zaffagnini M, and Trost P. Glutathionylation of cytosolic glyceraldehyde-3-phosphate dehydrogenase from the model plant Arabidopsis thaliana is reversed by both glutaredoxins and thioredoxins in vitro. Biochem J 445: 337-347, 2012.

14. Bernal-Bayard P, Ojeda V, Hervás M, Cejudo FJ, Navarro JA, Velázquez-Campoy A, and Pérez-Ruiz JM. Molecular recognition in the interaction of chloroplast 2-Cys peroxiredoxin with NADPH-thioredoxin reductase C (NTRC) and thioredoxin x. FEBS Lett 588: 4342-4347, 2014.

15. Bertoldi M. Human peroxiredoxins 1 and 2 and their interacting protein partners; through structure toward functions of biological complexes. Protein Peptide Lett 23: 69-77, 2016.

16. Biteau B, Labarre J, and Toledano MB. ATP-dependent reduction of cysteine-sulphinic acid by $S$. cerevisiae sulphiredoxin. Nature 425: 980-984, 2003.

17. Broin M, Cuiné S, Eymery F, and Rey P. The plastidic 2cysteine peroxiredoxin is a target for a thioredoxin involved 
in the protection of the photosynthetic apparatus against oxidative damage. Plant Cell 14: 1417-1432, 2002.

18. Burn P. Talking point Amphitropic proteins: a new class of membrane proteins. Trends Biochem Sci 13: 79-83, 1988.

19. Cao P, Jung KH, Choi D, Hwang D, Zhu J, and Ronald PC. The rice oligonucleotide array database: an atlas of rice gene expression. Rice 5: 17, 2012.

20. Cerveau D, Kraut A, Stotz HU, Mueller MJ, Couté Y, Rey P. Characterization of the Arabidopsis thaliana 2Cys peroxiredoxin interactome. Plant Sci 252: 30-41, 2016.

21. Cerveau D, Ouahrani D, Marok MA, Blanchard L, and Rey P. Physiological relevance of plant 2-Cys peroxiredoxin overoxidation level and oligomerization status. Plant Cell Environ 39: 103-119, 2016.

22. Cha MK, Yun $\mathrm{CH}$, and Kim IH. Interaction of human thiol-specific antioxidant protein 1 with erythrocyte plasma membrane. Biochemistry 39: 6944-6950, 2000.

23. Chae HZ, Chung SJ, and Rhee SG. Thioredoxindependent peroxide reductase from yeast. $J$ Biol Chem 269: 27670-27678, 1994.

24. Chen HH, Chu P, Zhou YL, Ding Y, Li Y, Liu J, Jiang LW, and Huang SZ. Ectopic expression of NnPER1, a Nelumbo nucifera 1-cysteine peroxiredoxin antioxidant, enhances seed longevity and stress tolerance in Arabidopsis. Plant J 88: 608-619, 2016.

25. Cho CS, Yoon HJ, Kim JY, Woo HA, and Rhee SG. Circadian rhythm of hyperoxidized peroxiredoxin II is determined by hemoglobin autoxidation and the $20 \mathrm{~S}$ proteasome in red blood cells. Proc Natl Acad Sci USA 111: 12043-12048, 2014.

26. Collin V, Issakidis-Bourguet E, Marchand C, Hirasawa M, Lancelin JM, Knaff DB, and Miginiac-Maslow M. The Arabidopsis plastidial thioredoxins New functions and new insights into specificity. J Biol Chem 278: $23747-$ 23752, 2003.

27. Dangoor I, Peled-Zehavi H, Wittenberg G, and Danon A. A chloroplast light-regulated oxidative sensor for moderate light intensity in Arabidopsis. Plant Cell 24: 18941906, 2012.

28. Dat J, Vandenabeele S, Vranová E, Van Montagu M, Inzé D, and Van Breusegem F. Dual action of the active oxygen species during plant stress responses. Cell Mol Life Sci 57: 779-795, 2000.

29. DeLano WL. The PyMOL molecular graphics system, 2002. DeLano Scientific, Palo Alto, CA. www.pymol .org (accessed July 5, 2017).

30. Dietz KJ. Redox signal integration: from stimulus to networks and genes. Physiol Plantarum 133: 459-468, 2008.

31. Dietz KJ. Peroxiredoxins in plants and cyanobacteria. Antioxid Redox Signal 15: 1129-1159, 2011.

32. Dietz KJ. Thiol-Based Peroxidases and Ascorbate Peroxidases: Why Plants Rely on Multiple Peroxidase Systems in the Photosynthesizing Chloroplast?. Mol Cells 39: 2025, 2016.

33. Dunkley TP, Hester S, Shadforth IP, Runions J, Weimar T, Hanton SL, and Watson RB. Mapping the Arabidopsis organelle proteome. Proc Natl Acad Sci USA 103: 65186523, 2006.

34. Edgar RS, Green EW, Zhao Y, van Ooijen G, Olmedo M, Qin X, Xu Y, Pan M, Valekunja UK, Feeney KA, Maywood ES, Hastings MH, Baliga NS, Merrow M, Millar AJ, Johnson CH, Kyriacou CP, O'Neill JS, and Reddy AB. Peroxiredoxins are conserved markers of circadian rhythms. Nature 485: 459-464, 2012.
35. Eliyahu E, Rog I, Inbal D, and Danon A. ACHT4-driven oxidation of APS1 attenuates starch synthesis under low light intensity in Arabidopsis plants. Proc Natl Acad Sci USA 112: 12876-12881, 2015.

36. Engelman R, Weisman-Shomer P, Ziv T, Xu J, Arnér ES, and Benhar M. Multilevel regulation of 2-Cys peroxiredoxin reaction cycle by S-nitrosylation. J Biol Chem 288: 11312-11324, 2013.

37. Engelsberger WR and Schulze WX. Nitrate and ammonium lead to distinct global dynamic phosphorylation patterns when resupplied to nitrogen-starved Arabidopsis seedlings. Plant J 69: 978-995, 2012.

38. Espunya MC, De Michele R, Gómez-Cadenas A, and Martínez MC. S-Nitrosoglutathione is a component of wound-and salicylic acid-induced systemic responses in Arabidopsis thaliana. J Exp Bot 63: 3219-3227, 2012.

39. Ferrer-Sueta G, Manta B, Botti H, Radi R, Trujillo M, and Denicola A. Factors affecting protein thiol reactivity and specificity in peroxide reduction. Chem Res Toxicol 24: 434-450, 2011.

40. Foyer $\mathrm{CH}$ and Noctor G. Ascorbate and glutathione: the heart of the redox hub. Plant Physiol 155: 2-18, 2011.

41. Gautier R, Douguet D, Antonny B, and Drin G. HELIQUEST: a web server to screen sequences with specific $\alpha$ helical properties. Bioinformatics 24, 2101-2102, 2008.

42. Gelhaye E, Rouhier N, Gérard J, Jolivet Y, Gualberto J, Navrot N, Ohlsson PI, Wingsle G, Hirasawa M, Knaff DB, Wang H, Dizengremel P, Meyer Y, and Jacquot JP. A specific form of thioredoxin $\mathrm{h}$ occurs in plant mitochondria and regulates the alternative oxidase. Proc Natl Acad Sci U S A 101: 14545-14550, 2004.

43. Ghosh S, Moore S, Bell RM, and Dush M. Functional analysis of a phosphatidic acid binding domain in human Raf-1 Kinase mutations in the phosphatidate binding domain lead to tail and trunk abnormalities in developing zebrafish embryos. J Biol Chem 278: 45690-45696, 2003.

44. Groitl B and Jakob U. Thiol-based redox switches. Biochim Biophys Acta 1844: 1335-1343, 2014.

45. Gutscher M, Sobotta MC, Wabnitz GH, Ballikaya S, Meyer AJ, Samstag Y, and Dick TP. Proximity-based protein thiol oxidation by $\mathrm{H} 2 \mathrm{O} 2$-scavenging peroxidases. J Biol Chem 284: 31532-31540, 2009.

46. Hall A, Karplus PA, and Poole LB. Typical 2-Cys peroxiredoxins-structures, mechanisms and functions. FEBS J 276: 2469-2477, 2009.

47. He D, Wang Q, Li M, Damaris RN, Yi X, Cheng Z, and Yang P. Global proteome analyses of lysine acetylation and succinylation reveal the widespread involvement of both modification in metabolism in the embryo of germinating rice seed. J Proteome Res 15: 879-890, 2016.

48. Heazlewood JL, Durek P, Hummel J, Selbig J, Weckwerth W, Walther D, and Schulze WX. PhosPhAt: a database of phosphorylation sites in Arabidopsis thaliana and a plantspecific phosphorylation site predictor. Nucleic Acids Res 36(Suppl 1): D1015-D1021, 2008.

49. Hofmann B, Hecht HJ, and Flohé L. Peroxiredoxins. Biol Chem 383: 347-364, 2002.

50. Hong SK, Cha MK, and Kim IH. Specific protein interaction of human Pag with Omi/HtrA2 and the activation of the protease activity of Omi/HtrA2. Free Radic Biol Med 40: 275-284, 2006.

51. Hossain MS, ElSayed AI, Moore M, and Dietz KJ. Redox and Reactive Oxygen Species Network in Acclimation for 
Salinity Tolerance in Sugar Beet. J Exp Bot 68: 12831298, 2017.

52. Huang C, Verrillo F, Renzone G, Arena S, Rocco M, Scaloni A, and Marra M. Response to biotic and oxidative stress in Arabidopsis thaliana: analysis of variably phosphorylated proteins. J Proteomics 74: 1934-1949, 2011.

53. Iglesias-Baena I, Barranco-Medina S, Sevilla F, and Lázaro JJ. The dual-targeted plant sulfiredoxin retroreduces the sulfinic form of atypical mitochondrial peroxiredoxin. Plant Physiol 155: 944-955, 2011.

54. Jang HH, Lee KO, Chi YH, Jung BG, Park SK, Park JH, Lee JR, Lee SS, Moon JC, Yun JW, Choi YO, Kim WY, Kang JS, Cheong GW, Yun DJ, Rhee SG, Cho MJ, and Lee SY. Two enzymes in one: two yeast peroxiredoxins display oxidative stress-dependent switching from a peroxidase to a molecular chaperone function. Cell 117: 625-635, 2004.

55. Johnson JE and Cornell RB. Amphitropic proteins: regulation by reversible membrane interactions (review). Mol Membr Biol 16: 217-235, 1999.

56. Jönsson TJ, Johnson LC, and Lowther WT. Structure of the sulphiredoxin-peroxiredoxin complex reveals an essential repair embrace. Nature 451: 98-101, 2008.

57. Kelley LA, Mezulis S, Yates CM, Wass MN, and Sternberg MJ. The Phyre2 web portal for protein modeling, prediction and analysis. Nat Protoc 10: 845-858, 2015.

58. König J, Baier M, Horling F, Kahmann U, Harris G, Schürmann P, and Dietz KJ. The plant-specific function of 2-Cys peroxiredoxin-mediated detoxification of peroxides in the redox-hierarchy of photosynthetic electron flux. Proc Natl Acad Sci USA 99: 5738-5743, 2002.

59. König J, Galliardt H, Jütte P, Schäper S, Dittmann L, and Dietz KJ. The conformational bases for the two functionalities of 2-cysteine peroxiredoxins as peroxidase and chaperone. J Exp Bot 64: 3483-3497, 2013.

60. König J, Lotte K, Plessow R, Brockhinke A, Baier M, and Dietz KJ. Reaction mechanism of plant 2-Cys peroxiredoxin role of the $\mathrm{C}$ terminus and the quaternary structure. J Biol Chem 278: 24409-24420, 2003.

61. Lemaire SD, Michelet L, Zaffagnini M, Massot V, and Issakidis-Bourguet E. Thioredoxins in chloroplasts. Curr Genet 51: 343-365, 2007.

62. Liebthal M, Strüve M, Li X, Hertle Y, Maynard D, Hellweg T, Viehhauser A, and Dietz KJ. Redoxdependent conformational dynamics of decameric 2cysteine peroxiredoxin and its interaction with cyclophilin 20-23. Plant Cell Physiol 57: 1415-1425, 2016.

63. Lindermayr C, Saalbach G, and Durner J. Proteomic identification of S-nitrosylated proteins in Arabidopsis. Plant Physiol 137: 921-930, 2005.

64. Manevich Y, Feinstein SI, and Fisher AB. Activation of the antioxidant enzyme 1-CYS peroxiredoxin requires glutathionylation mediated by heterodimerization with

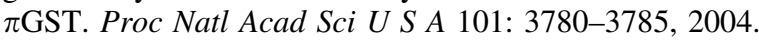

65. Manevich Y, Reddy KS, Shuvaeva T, Feinstein SI, and Fisher AB. Structure and phospholipase function of peroxiredoxin 6: identification of the catalytic triad and its role in phospholipid substrate binding. J Lipid Res 48: 2306-2318, 2007.

66. Manta B, Hugo M, Ortiz C, Ferrer-Sueta G, Trujillo M, and Denicola A. The peroxidase and peroxynitrite reductase activity of human erythrocyte peroxiredoxin 2 . Arch Biochem Biophys 484: 146-154, 2009.

67. Margaria P, Abbà S, and Palmano S. Novel aspects of grapevine response to phytoplasma infection investigated by a proteomic and phospho-proteomic approach with data integration into functional networks. BMC Genomics 14: 1, 2013.

68. Matte A, Bertoldi M, Mohandas N, An X, Bugatti A, Brunati AM, Rusnati M, Tibaldi E, Siciliano A, Turrini F, Perrotta S, and De Franceschi L. Membrane association of peroxiredoxin-2 in red cells is mediated by the N-terminal cytoplasmic domain of band 3. Free Radic Biol Med 55: 27-35, 2013.

69. Michelet L, Zaffagnini M, Marchand C, Collin V, Decottignies $\mathrm{P}$, Tsan $\mathrm{P}$, Lancelin JM, Trost $\mathrm{P}$, MiginiacMaslow M, Noctor G, and Lemaire SD. Glutathionylation of chloroplast thioredoxin $\mathrm{f}$ is a redox signaling mechanism in plants. Proc Natl Acad Sci U S A 102: 1647816483, 2005.

70. Mithoe SC, Boersema PJ, Berke L, Snel B, Heck AJ, and Menke FL. Targeted quantitative phosphoproteomics approach for the detection of phospho-tyrosine signaling in plants. J Proteome Res 11: 438-448, 2012.

71. Mock HP, and Dietz KJ. Redox proteomics for the assessment of redox-related posttranslational regulation in plants. Biochim Biophys Acta 1864: 967-973, 2016.

72. Monteiro G, Horta BB, Pimenta DC, Augusto O, and Netto LE. Reduction of 1-Cys peroxiredoxins by ascorbate changes the thiol-specific antioxidant paradigm, revealing another function of vitamin C. Proc Natl Acad Sci USA 104: 4886-4891, 2007.

73. Moore RB and Shriver SK. Protein $7.2 \mathrm{~b}$ of human erythrocyte membranes binds to calpromotin. Biochem Biophys Res Commun 232, 294-297, 1997.

74. Moore RB, Mankad MV, Shriver SK, Mankad VN, and Plishker GA. Reconstitution of $\mathrm{Ca}(2+)$-dependent $\mathrm{K}+$ transport in erythrocyte membrane vesicles requires a cytoplasmic protein. J Biol Chem 266, 18964-18968, 1991.

75. Morais MA, Giuseppe PO, Souza TA, Alegria TG, Oliveira MA, Netto LE, and Murakami MT. How pH modulates the dimer-decamer interconversion of 2-Cys peroxiredoxins from the Prx1 subfamily. J Biol Chem 290: 8582-8590, 2015.

76. Muthuramalingam M, Matros A, Scheibe R, Mock HP, and Dietz KJ. The hydrogen peroxide-sensitive proteome of the chloroplast in vitro and in vivo. Front Plant Sci 4: 54, 2013.

77. Muthuramalingam M, Seidel T, Laxa M, De Miranda SMN, Gärtner F, Ströher E, Kandlbinder A, and Dietz KJ. Multiple redox and non-redox interactions define 2-Cys peroxiredoxin as a regulatory hub in the chloroplast. Mol Plant 2: 1273-1288, 2009.

78. Nakagami H, Sugiyama N, Mochida K, Daudi A, Yoshida Y, Toyoda T, Tomita M, Ishihama Y, and Shirasu K. Large-scale comparative phosphoproteomics identifies conserved phosphorylation sites in plants. Plant Physiol 153: 1161-1174, 2010.

79. Nietzel T, Mostertz J, Hochgräfe F, and Schwarzländer M. Redox regulation of mitochondrial proteins and proteomes by cysteine thiol switches. Mitochondrion 33: 72-83, 2016.

80. Noctor G, Mhamdi A, Chaouch S, Han YI, Neukermans J, Marquez-Garcia B, Queval G, and Foyer CH. Glutathione in plants: an integrated overview. Plant Cell Environ 35: 454-484, 2012.

81. Noguera-Mazon V, Krimm I, Walker O, and Lancelin JM. Protein-protein interactions within peroxiredoxin systems. Photosynthesis Res 89: 277-290, 2006. 
82. Noichri Y, Palais G, Ruby V, D'Autreaux B, DelaunayMoisan A, Nyström T, Molin M, and Toledano MB. In vivo parameters influencing 2-CysPrx oligomerization: the role of enzyme sulfinylation. Redox Biol 6: 326-333, 2015.

83. Pan Y, Jin JH, Yu Y, and Wang J. Significant enhancement of hPrx1 chaperone activity through lysine acetylation. Chembiochem 15: 1773-1776, 2014.

84. Park JW, Piszczek G, Rhee SG, and Chock PB. Glutathionylation of peroxiredoxin I induces decamer to dimers dissociation with concomitant loss of chaperone activity. Biochemistry 50: 3204-3210, 2011.

85. Park SW, Li W, Viehhauser A, He B, Kim S, Nilsson AK, Andersson MX, Kittle JD, Ambavaram MM, Luan S, Esker AR, Tholl D, Cimini D, Ellerstrom M, Coaker G, Mitchell TK, Pereira A, Dietz KJ, and Lawrence CB. Cyclophilin 20-3 relays a 12-oxo-phytodienoic acid signal during stress responsive regulation of cellular redox homeostasis. Proc Natl Acad Sci USA 110: 9559-9564, 2013.

86. Parsonage D, Youngblood DS, Sarma GN, Wood ZA, Karplus PA, and Poole LB. Analysis of the Link between Enzymatic Activity and Oligomeric State in AhpC, a Bacterial Peroxiredoxin $\uparrow$. Biochemistry 44: 10583-10592, 2005.

87. Pedrajas JR, McDonagh B, Hernández-Torres F, MirandaVizuete A, González-Ojeda R, Martínez-Galisteo E, Padilla CA, and Bárcena JA. Glutathione is the resolving thiol for thioredoxin peroxidase activity of 1-Cys peroxiredoxin without being consumed during the catalytic cycle. Antioxid Redox Signal 24: 115-128, 2016.

88. Pehar M, Ball LE, Sharma DR, Harlan BA, ComteWalters S, Neely BA, and Vargas MR. Changes in protein expression and lysine acetylation induced by decreased glutathione levels in astrocytes. Mol Cell Proteomics 15: 493-505, 2015.

89. Peltier JB, Cai Y, Sun Q, Zabrouskov V, Giacomelli L, Rudella A, Ytterberg AJ, Rutschow H, and van Wijk KJ. The oligomeric stromal proteome of Arabidopsis thaliana chloroplasts. Mol Cell Proteomics 5: 114-133, 2006.

90. Pérez-Ruiz JM, Spínola MC, Kirchsteiger K, Moreno J, Sahrawy M, and Cejudo FJ. Rice NTRC is a highefficiency redox system for chloroplast protection against oxidative damage. Plant Cell 18: 2356-2368, 2006.

91. Perkins A, Nelson KJ, Parsonage D, Poole LB, and Karplus PA. Peroxiredoxins: guardians against oxidative stress and modulators of peroxide signaling. Trends Biochem Sci 40: 435-445, 2015.

92. Plishker GA, Chevalier D, Seinsoth L, and Moore RB. Calcium-activated potassium transport and high molecular weight forms of calpromotin. J Biol Chem 267: 2183921843, 1992.

93. Poole LB. The basics of thiols and cysteines in redox biology and chemistry. Free Radic Biol Med 80: 148-157, 2015.

94. Puerto-Galán L, Pérez-Ruiz JM, Guinea M, and Cejudo FJ. The contribution of NADPH thioredoxin reductase $\mathrm{C}$ (NTRC) and sulfiredoxin to 2-Cys peroxiredoxin overoxidation in Arabidopsis thaliana chloroplasts. J Exp Bot 66: 2957-2966, 2015.

95. Pulido P, Cazalis R, and Cejudo FJ. An antioxidant redox system in the nucleus of wheat seed cells suffering oxidative stress. Plant J 57: 132-145, 2009.

96. Pulido P, Spínola MC, Kirchsteiger K, Guinea M, Pascual MB, Sahrawy M, Sandalio LM, Dietz KJ, González M, and Cejudo FJ. Functional analysis of the pathways for 2Cys peroxiredoxin reduction in Arabidopsis thaliana chloroplasts. J Exp Bot 61: 4043-4054, 2010.

97. Qi Y and Grishin NV. Structural classification of thioredoxin-like fold proteins. Proteins 58: 376-388, 2005.

98. Reiland S, Finazzi G, Endler A, Willig A, Baerenfaller K, Grossmann J, and Baginsky S. Comparative phosphoproteome profiling reveals a function of the STN8 kinase in fine-tuning of cyclic electron flow (CEF). Proc Natl Acad Sci USA 108: 12955-12960, 2011.

99. Reiland S, Messerli G, Baerenfaller K, Gerrits B, Endler A, Grossmann J, Gruissem W, and Baginsky S. Largescale Arabidopsis phosphoproteome profiling reveals novel chloroplast kinase substrates and phosphorylation networks. Plant Physiol 150: 889-903, 2009.

100. Rhee SG. Overview on Peroxiredoxin. Mol Cells 39: 1-5, 2016a.

101. Rhee SG and Kil IS. Mitochondrial $\mathrm{H}_{2} \mathrm{O}_{2}$ signaling is controlled by the concerted action of peroxiredoxin III and sulfiredoxin: linking mitochondrial function to circadian rhythm. Free Radic Biol Med 99: 120-127, 2016.

102. Rochaix JD. Redox regulation of thylakoid protein kinases and photosynthetic gene expression. Antioxid Redox Signal 18: 2184-2201, 2013.

103. Roitinger E, Hofer M, Köcher T, Pichler P, Novatchkova M, Yang J, Schlögelhofer P, and Mechtler K. Quantitative phosphoproteomics of the ataxia telangiectasia-mutated (ATM) and ataxia telangiectasia-mutated and rad3-related (ATR) dependent DNA damage response in Arabidopsis thaliana. Mol Cell Proteomics 14: 556-571, 2015.

104. Romero-Puertas MC, Laxa M, Mattè A, Zaninotto F, Finkemeier I, Jones AM, Perazzolli M, Vandelle E, Dietz $\mathrm{KJ}$, and Delledonne M. S-nitrosylation of peroxiredoxin II E promotes peroxynitrite-mediated tyrosine nitration. Plant Cell 19: 4120-4130, 2007.

105. Rose CM, Venkateshwaran M, Volkening JD, Grimsrud PA, Maeda J, Bailey DJ, and Westphall MS. Rapid phosphoproteomic and transcriptomic changes in the rhizobia-legume symbiosis. Mol Cell Proteomics 11: 724744, 2012.

106. Rouhier N, Gelhaye E, Sautiere PE, Brun A, Laurent P, Tagu D, Gerard J, de Fay E, Meyer Y, and Jacquot JP. Isolation and characterization of a new peroxiredoxin from poplar sieve tubes that uses either glutaredoxin or thioredoxin as a proton donor. Plant Physiol 127: 1299-1309, 2001.

107. Sakamoto A, Tsukamoto S, Yamamoto H, UedaHashimoto M, Takahashi M, Suzuki H, and Morikawa H. Functional complementation in yeast reveals a protective role of chloroplast 2-Cys peroxiredoxin against reactive nitrogen species. Plant J 33: 841-851, 2003.

108. Schwacke R, Schneider A, van der Graaff E, Fischer K, Catoni E, Desimone M, and Kunze R. ARAMEMNON, a novel database for Arabidopsis integral membrane proteins. Plant Physiol 131: 16-26, 2003.

109. Seo JH, Lim JC, Lee DY, Kim KS, Piszczek G, Nam HW, Kim YS, Ahn T, Yun CH, Kim K, Chock PB, and Chae HS. Novel protective mechanism against irreversible hyperoxidation of peroxiredoxin $\mathrm{N} \alpha$-terminal acetylation of human peroxiredoxin II. J Biol Chem 284: 13455-13465, 2009.

110. Sevilla F, Camejo D, Ortiz-Espín A, Calderón A, Lázaro JJ, and Jiménez A. The thioredoxin/peroxiredoxin/sulfiredoxin system: current overview on its redox function in plants and regulation by reactive oxygen and nitrogen species. J Exp Bot 66: 2945-2955, 2015. 
111. Soito L, Williamson C, Knutson ST, Fetrow JS, Poole LB, and Nelson KJ. PREX: PeroxiRedoxin classification indEX, a database of subfamily assignments across the diverse peroxiredoxin family. Nucleic Acids Res 39(Suppl 1): D332-D337, 2011.

112. Speiser A, Haberland S, Watanabe M, Wirtz M, Dietz KJ, Saito K, and Hell R. The significance of cysteine synthesis for acclimation to high light conditions. Front Plant Sci 5: 776, 2015.

113. Stacy RA, Munthe E, Steinum T, Sharma B, and Aalen RB. A peroxiredoxin antioxidant is encoded by a dormancy-related gene, Per1, expressed during late development in the aleurone and embryo of barley grains. Plant Mol Biol 31: 1205-1216, 1996.

114. Umezawa T, Sugiyama N, Takahashi F, Anderson JC, Ishihama Y, Peck SC, and Shinozaki K. Genetics and phosphoproteomics reveal a protein phosphorylation network in the abscisic acid signaling pathway in Arabidopsis thaliana. Sci Signal 6: rs8, 2013.

115. van Wijk KJ, Friso G, Walther D, and Schulze WX. Metaanalysis of Arabidopsis thaliana phospho-proteomics data reveals compartmentalization of phosphorylation motifs. Plant Cell 26: 2367-2389, 2014.

116. Wang P, Xue L, Batelli G, Lee S, Hou YJ, Van Oosten MJ, Zhang H, Tao WA, and Zhu JK. Quantitative phosphoproteomics identifies SnRK2 protein kinase substrates and reveals the effectors of abscisic acid action. Proc Natl Acad Sci USA 110: 11205-11210, 2013.

117. Wang X, Bian Y, Cheng K, Zou H, Sun SSM, and He JX. A comprehensive differential proteomic study of nitrate deprivation in Arabidopsis reveals complex regulatory networks of plant nitrogen responses. J Proteome Res 11: 2301-2315, 2012.

118. Woo HA, Jeong W, Chang TS, Park KJ, Park SJ, Yang JS, and Rhee SG. Reduction of cysteine sulfinic acid by sulfiredoxin is specific to 2-cys peroxiredoxins. $J$ Biol Chem 280: 3125-3128, 2005.

119. Woo HA, Yim SH, Shin DH, Kang D, Yu DY, and Rhee SG. Inactivation of peroxiredoxin I by phosphorylation allows localized $\mathrm{H}_{2} \mathrm{O}_{2}$ accumulation for cell signaling. Cell 140: 517-528, 2010.

120. Wood ZA, Poole LB, Hantgan RR, and Karplus PA. Dimers to Doughnuts: Redox-Sensitive Oligomerization of 2-Cysteine Peroxiredoxins†. Biochemistry 41: 5493-5504, 2002.

121. Wood ZA, Poole LB, and Karplus PA. Peroxiredoxin evolution and the regulation of hydrogen peroxide signaling. Science 300: 650-653, 2003a.

122. Wood ZA, Schröder E, Harris JR, and Poole LB. Structure, mechanism and regulation of peroxiredoxins. Trends Biochem Sci 28: 32-40, 2003b.

123. Wu XN, Rodriguez CS, Pertl-Obermeyer H, Obermeyer $\mathrm{G}$, and Schulze WX. Sucrose-induced receptor kinase SIRK1 regulates a plasma membrane aquaporin in Arabidopsis. Mol Cell Proteomics 12: 2856-2873, 2013.

124. Xiong Y, Zhang K, Cheng Z, Wang GL, and Liu W. Data for global lysine-acetylation analysis in rice (Oryza sativa). Data Brief 7: 411-417, 2016.

125. Xu S, Ding H, Su F, Zhang A, and Jiang M. Involvement of Protein Phosphorylation in Water Stress-induced Antioxidant Defense in Maize Leaves. J Integr Plant Biol 51: 654-662, 2009.
126. Yang Z, Guo G, Zhang M, Liu CY, Hu Q, Lam H, and Li N. Stable isotope metabolic labeling-based quantitative phosphoproteomic analysis of Arabidopsis mutants reveals ethylene-regulated time-dependent phosphoproteins and putative substrates of constitutive triple response 1 kinase. Mol Cell Proteomics 12: 3559-3582, 2013.

127. Yao Q, Ge H, Wu S, Zhang N, Chen W, Xu C, Gao J, Thelen JJ, and Xu D. P3DB 3.0: from plant phosphorylation sites to protein networks. Nucleic Acids Res 42: D1206-D1213, 2013.

128. Zhang H, Zhou H, Berke L, Heck AJ, Mohammed S, Scheres B, and Menke F. L. Quantitative phosphoproteomics after auxin-stimulated lateral root induction identifies an SNX1 protein phosphorylation site required for growth. Mol Cell Proteomics 12: 1158-1169, 2013.

Address correspondence to: Prof. Karl-Josef Dietz.

Department of Biochemistry and Physiology of Plants Faculty of Biology University of Bielefeld

University Street 25

Bielefeld D-33501

Germany

E-mail: karl-josef.dietz@uni-bielefeld.de

Date of first submission to ARS Central, May 10, 2017; date of acceptance, June 2, 2017.

\begin{tabular}{|c|}
\hline $\begin{aligned} & \text { Abbreviations Used } \\
\text { 2-CysPrx } & =2 \text {-cysteine peroxiredoxin } \\
\mathrm{APR} & =\text { adenylylsulfate reductase } \\
\mathrm{Apx} & =\text { ascorbate peroxidase } \\
\mathrm{CoIP} & =\text { coimmuno precipitation } \\
\mathrm{CTC} & =\text { critical transition concentration } \\
\mathrm{Cyp} 20-3 & =\text { cyclophilin } 20-3 \\
\mathrm{Fd} & =\text { ferredoxin } \\
\mathrm{Gpx} & =\text { glutathione peroxidase } \\
\mathrm{GSH} / \mathrm{GSSG} & =\text { reduced/oxidized glutathione } \\
\mathrm{GSNO} & =\text { nitrosoglutathione } \\
\mathrm{GST} & =\text { glutathione- } S \text {-transferase } \\
\mathrm{HMW} & =\text { high molecular weight } \\
\mathrm{ITC} & =\text { isothermal titration microcalorimetry } \\
\mathrm{NTRC} & =\text { NADPH-dependent thioredoxin } \\
\text { OPDA } & =\text { rexophytodienoic acid } \\
\mathrm{PAGE} & =\text { polyacrylamide gel electrophoresis } \\
\mathrm{PET} & =\text { photosynthetic electron transport } \\
\text { Prx/Prdx } & =\text { peroxiredoxin } \\
\mathrm{PTMs} & =\text { post-translational modifications } \\
\mathrm{ROS} & =\text { reactive oxygen species } \\
\mathrm{sApx} & =\text { stromal ascorbate peroxidase } \\
\mathrm{SEC} & =\text { size exclusion chromatography } \\
\mathrm{SOD} & =\text { superoxide dismutase } \\
\mathrm{Srx} & =\text { sulfiredoxin } \\
\mathrm{tApx} & =\text { thylakoid-bound peroxidase } \\
\mathrm{Trx} & =\text { thioredoxin }\end{aligned}$ \\
\hline
\end{tabular}

